\title{
Syntheses, Structure, and Optical Properties of Ladder-Type Fused Azaborines
}

Tomohiro Agou, Junji Kobayashi, and Takayuki Kawashima*

Department of Chemistry, Graduate School of Science, The University of Tokyo

7-3-1 Hongo, Bunkyo-ku, Tokyo 113-0033, Japan

takayuki@chem.s.u-tokyo.ac.jp

\section{Experimental Section}

General Procedure. General chemicals were used as received. Cyclohexane for spectrochemical or fluorometric grade (Dojindo) was used for optical measurements. All manipulations were carried out using standard Schlenk technique under an argon atmosphere. Solvents were purified by MBRAUN MB-SPS system. Wet column chromatography (WCC) was performed using Kanto Silica Gel 60N. Gel permeation liquid chromatography (GPLC) was performed using LC-918 or LC-908 with JAIGEL $1 \mathrm{H}+2 \mathrm{H}$ columns (Japan Analytical Industry) using chloroform or toluene as solvents, respectively. NMR spectra were recorded on a JEOL AL-400 spectrometer $\left({ }^{1} \mathrm{H}, 400 \mathrm{MHz} ;{ }^{13} \mathrm{C}, 100\right.$ MHz; $\left.{ }^{11} \mathrm{~B}, 128 \mathrm{MHz}\right)$, a JEOL ECX-400 spectrometer $\left({ }^{1} \mathrm{H}, 400 \mathrm{MHz},{ }^{13} \mathrm{C}, 100 \mathrm{MHz}\right)$, a Bruker DRX-500 spectrometer $\left({ }^{1} \mathrm{H}, 500 \mathrm{MHz} ;{ }^{13} \mathrm{C}, 126 \mathrm{MHz}\right)$, or a Bruker AV-500 spectrometer $\left({ }^{1} \mathrm{H}, 500\right.$ $\mathrm{MHz},{ }^{13} \mathrm{C}, 126 \mathrm{MHz}$ ). Chemical shifts are reported in $\delta .{ }^{1} \mathrm{H}$ NMR spectra are referenced to residual protons in deuterated solvent; ${ }^{13} \mathrm{C}$ NMR spectra are referenced to carbon-13 in the deuterated solvent; ${ }^{11} \mathrm{~B}$ NMR spectra are referenced to an external standard of $\mathrm{BF}_{3} \bullet \mathrm{Et}_{2} \mathrm{O}$. Low resolution mass spectra were obtained with a JEOL JMS-700P. High resolution mass spectra were recorded on a JEOL JMS-700P using $m$-nitrobenzylalchol as matrix and PEG400, PEG600, or Ultramark ${ }^{\circledR}$ as standards. UV-vis spectra were recorded on a JASCO V-530 spectrophotometer. Steady-state fluorescence spectra were recorded on a HITACHI F4500 fluorescence spectrophotometer. All melting points were measured with a Yanaco MP-S3 and uncorrected. Elemental analyses were performed by the Microanalytical Laboratory of Department of Chemistry, Faculty of Science, the University of Tokyo. N,N-bis(2-bromo-4-methylphenyl)methylamine ${ }^{1}$, 1,4-dibromo-2,5-diiodobenzene and 1,3-dibromo-4,6-diiodobenzene ${ }^{2}$ were synthesized according to literatures.

\section{2-Bromo-4-butylaniline}

This compound was synthesized using a reported procedure. ${ }^{3}$

A mixture of 4-butylaniline ( $37 \mathrm{~g}, 0.25 \mathrm{~mol}), \mathrm{NaVO}_{3}(3.1 \mathrm{~g}, 25 \mathrm{mmol}), \mathrm{KBr}(33 \mathrm{~g}, 0.28 \mathrm{~mol})$, $\mathrm{NaBO}_{3} \cdot 4 \mathrm{H}_{2} \mathrm{O}(39 \mathrm{~g}, 0.25 \mathrm{~mol})$ and $\mathrm{AcOH}(200 \mathrm{~mL})$ was stirred at r.t. for $4 \mathrm{~h}$ under air. The mixture was neutralized with aqueous $\mathrm{Na}_{2} \mathrm{CO}_{3}$, and the aqueous layer was extracted with $\mathrm{Et}_{2} \mathrm{O}$. The organic 
layer was washed by aqueous $\mathrm{Na}_{2} \mathrm{SO}_{3}$ and $\mathrm{H}_{2} \mathrm{O}$ and dried over $\mathrm{Na}_{2} \mathrm{SO}_{4}$. Crude product was purified by distillation $\left(0.2 \mathrm{mmHg}, 97^{\circ} \mathrm{C}\right)(50 \mathrm{~g}, 88 \%)$.

Pale brown liquid, ${ }^{1} \mathrm{H}$ NMR (400 MHz, $\left.\mathrm{CDCl}_{3}\right) \delta 0.90$ (t, $J=7.2 \mathrm{~Hz}, 3 \mathrm{H}$ ), 1.32 (sext, $J=7.4 \mathrm{~Hz}$, $2 \mathrm{H}$ ), 1.53 (quint, $J=7.6 \mathrm{~Hz}, 2 \mathrm{H}$ ), 2.47 (t, $J=7.6 \mathrm{~Hz}, 2 \mathrm{H}$ ), 3.93 (brs, $2 \mathrm{H}$ ), 6.68 (d, $J=8.0 \mathrm{~Hz}, 1 \mathrm{H}$ ), $6.91(\mathrm{dd}, J=8.0,2.0 \mathrm{~Hz}, 1 \mathrm{H}), 7.22(\mathrm{~d}, J=2.0 \mathrm{~Hz}, 1 \mathrm{H}) ;{ }^{13} \mathrm{C} \mathrm{NMR}\left(126 \mathrm{MHz}, \mathrm{CDCl}_{3}\right) \delta 13.82,22.08$, 33.60, 34.21, 109.11, 115.61, 128.21, 131.91, 134.06, 141.57; HRMS $\left(\mathrm{FAB}^{+}\right) \mathrm{m} / \mathrm{z}$ calcd for $\mathrm{C}_{10} \mathrm{H}_{14} \mathrm{~N}^{79} \mathrm{Br} 227.0320$, found: 227.0315, calcd for $\mathrm{C}_{10} \mathrm{H}_{14} \mathrm{~N}^{81} \mathrm{Br} 229.0290$, found: 229.0315 .

\section{3,6,9-Trimethyl-10-mesityl-9,10-dihydro-9-aza-10-boraanthracene (9)}

To a $\mathrm{Et}_{2} \mathrm{O}$ solution $(50 \mathrm{~mL})$ of $N, N$-bis(2-bromo-4-methylphenyl)methylamine $(0.50 \mathrm{~g}, 1.4 \mathrm{mmol})$ at $-75^{\circ} \mathrm{C}$ was added $t$-BuLi $(2.2 \mathrm{M}$ in pentane, $3.2 \mathrm{~mL}, 7.0 \mathrm{mmol})$, and the mixture was stirred for 30 minutes at $0{ }^{\circ} \mathrm{C}$. After addition of $\mathrm{MesB}(\mathrm{OMe})_{2}(0.38 \mathrm{~mL}, 1.8 \mathrm{mmol})$, the reaction mixture was refluxed for $12 \mathrm{~h}$. The resulting suspension was filtered by Celite ${ }^{\circledR}$ pad, and the solvents were evaporated. The crude product was separated by GPLC to give 9 as yellow solids ( $0.37 \mathrm{~g}, 79 \%)$.

9: yellow solids, mp 236-239 ${ }^{\circ} \mathrm{C}$ (dec.). ${ }^{1} \mathrm{H}$ NMR (500 MHz, $\left.\mathrm{CDCl}_{3}\right) \delta 1.96$ (s, 6H), 2.35 (s, 6H), $2.42(\mathrm{~s}, 3 \mathrm{H}), 4.02(\mathrm{~s}, 3 \mathrm{H}), 6.96(\mathrm{~s}, 2 \mathrm{H}), 7.55-7.60(\mathrm{~m}, 6 \mathrm{H}) ;{ }^{13} \mathrm{C} \mathrm{NMR}\left(126 \mathrm{MHz}, \mathrm{CDCl}_{3}\right) \delta 20.52$, 21.34, 23.26, 35.39, 114.61, 126.67, 126.92, 128.24, 134.69, 136.03, 136.51, 136.78, 139.31, 144.42; ${ }^{11} \mathrm{~B}$ NMR $\left(128 \mathrm{MHz}, \mathrm{CDCl}_{3}\right) \delta 54.8\left(h_{1 / 2}=1200 \mathrm{~Hz}\right)$; HRMS $\left(\mathrm{FAB}^{+}\right) \mathrm{m} / \mathrm{z}$ calcd for $\mathrm{C}_{24} \mathrm{H}_{26} \mathrm{~N}^{10} \mathrm{~B}$ 338.2165, found: 338.2134; UV-vis (cyclohexane) $\lambda_{\max } / \mathrm{nm}(\log \varepsilon): 405$ (4.02), 386 (3.86), 258 (4.83).

\section{2,5-Dibromo- $N, N$ '-bis(2-bromo-4-butylphenyl)- $N, N$ '-dimethylbenzene-1,4-diamine (1)}

A mixture of 2-bromo-4-butylaniline (4.6 g, $20 \mathrm{mmol})$, 1,4-dibromo-2,5-diiodobenzene (5.0 g, 10 $\mathrm{mmol}), \mathrm{Pd}(\mathrm{dba})_{2}(0.23 \mathrm{~g}, 0.40 \mathrm{mmol})$, DPPF (0.44 g, $\left.0.79 \mathrm{mmol}\right), t-\mathrm{BuONa}(2.9 \mathrm{~g}, 30 \mathrm{mmol})$ and toluene $(100 \mathrm{~mL})$ was stirred at $80{ }^{\circ} \mathrm{C}$ for $12 \mathrm{~h}$. To this reaction mixture was added aqueous $\mathrm{NH}_{4} \mathrm{Cl}$, and the aqueous layer was extracted with $\mathrm{Et}_{2} \mathrm{O}$. The organic layer was dried with $\mathrm{Na}_{2} \mathrm{SO}_{4}$, and the solvents were evaporated. The crude product was separated by GPLC, and recrystallization from $\mathrm{CHCl}_{3} / \mathrm{EtOH}$ give 2,5-Dibromo- $N, N^{\prime}$-bis(2-bromo-4-butylphenyl)benzene-1,4-diamine (10) as tan solids (3.1 g, 45\%). To a THF solution $(80 \mathrm{~mL})$ of $\mathbf{1 0}(3.1 \mathrm{~g}, 4.5 \mathrm{mmol})$ at $-75^{\circ} \mathrm{C}$ was added MeLi (1.0 $\mathrm{M}$ in $\left.\mathrm{Et}_{2} \mathrm{O}, 15 \mathrm{~mL}, 15 \mathrm{mmol}\right)$. After the mixture was stirred for 30 minutes, MeI $(4.2 \mathrm{~mL}, 67$ mmol) was added, and the mixture was warmed to r.t. and stirred for $10 \mathrm{~h}$. After addition of water, the aqueous layer was extracted with $\mathrm{CHCl}_{3}$, and the organic layer was dried with $\mathrm{Na}_{2} \mathrm{SO}_{4}$. After evaporation of the solvents, the crude product was subjected to column chromatography $\left(\mathrm{SiO}_{2}\right.$, eluant: $\mathrm{CHCl}_{3}$ ) to give $\mathbf{1}$ as colorless solids (2.59 g, 36\% (2 steps)).

10: pale brown solids. ${ }^{1} \mathrm{H}$ NMR $\left(500 \mathrm{MHz}, \mathrm{CDCl}_{3}\right) \delta 0.93(\mathrm{t}, J=7.3 \mathrm{~Hz}, 6 \mathrm{H}), 1.35$ (sext, $J=7.4 \mathrm{~Hz}$, 4H), 1.57 (q, $J=7.6 \mathrm{~Hz}, 4 \mathrm{H}), 2.54$ (t, $J=7.7 \mathrm{~Hz}, 4 \mathrm{H}), 7.03-7.09$ (m, 4H), 7.39 (d, $J=0.9 \mathrm{~Hz}, 2 \mathrm{H})$, 
$7.43(\mathrm{~s}, 2 \mathrm{H}) ;{ }^{13} \mathrm{C}$ NMR $\left(126 \mathrm{MHz}, \mathrm{CDCl}_{3}\right) \delta 13.93,22.26,33.56,34.57,113.93,114.11,117.85$, 122.54, 128.32, 132.86, 135.42, 137.77 (Two signals are overlapped.); LRMS (FAB $\left.{ }^{+}\right): m / z 684\left(\mathrm{M}^{+}\right)$. 1: colorless solids, mp 130-132 ${ }^{\circ} \mathrm{C} .{ }^{1} \mathrm{H}$ NMR $\left(500 \mathrm{MHz}, \mathrm{CDCl}_{3}\right) \delta 0.91(\mathrm{t}, J=7.3 \mathrm{~Hz}, 6 \mathrm{H}), 1.33$ (sext, $J=7.4 \mathrm{~Hz}, 4 \mathrm{H}), 1.56$ (q, $J=7.7 \mathrm{~Hz}, 4 \mathrm{H}), 2.53$ (t, $J=7.7 \mathrm{~Hz}, 4 \mathrm{H}), 3.15(\mathrm{~s}, 6 \mathrm{H}), 6.86$ (d, $J=8.4$ $\mathrm{Hz}, 2 \mathrm{H}), 7.03$ (dd, $J=8.4,1.9 \mathrm{~Hz}, 2 \mathrm{H}), 7.16(\mathrm{~s}, 2 \mathrm{H}), 7.37$ (d, $J=1.9 \mathrm{~Hz}, 2 \mathrm{H}) ;{ }^{13} \mathrm{C}$ NMR $(126 \mathrm{MHz}$, $\left.\mathrm{CDCl}_{3}\right) \delta 13.91,22.26,33.35,34.57,41.52,118.85,120.23,123.67,128.18,128.32,134.03,140.21$, 145.01, 145.66; LRMS (FAB $\left.{ }^{+}\right): m / z 712\left(\mathrm{M}^{+}\right)$. Anal. Calcd for $\mathrm{C}_{28} \mathrm{H}_{32} \mathrm{Br}_{4} \mathrm{~N}_{2}: \mathrm{C}, 46.96 ; \mathrm{H}, 4.50 ; \mathrm{N}$, 3.91. Found: C, 46.83; H, 4.53; N, 3.72 .

\section{4,6-Dibromo- $N, N^{\prime}$-bis(2-bromo-4-butylphenyl)- $N, N$ '-dimethylbenzene-1,3-diamine (2)}

A mixture of 2-bromo-4-butylaniline (4.6 g, $20 \mathrm{mmol})$, 1,3-dibromo-4,6-diiodobenzene (4.8 g, 10 $\mathrm{mmol}), \operatorname{Pd}(\mathrm{dba})_{2}(0.58 \mathrm{~g}, 1.0 \mathrm{mmol})$, DPPF $(0.83 \mathrm{~g}, 1.5 \mathrm{mmol}), t-\mathrm{BuONa}(2.9 \mathrm{~g}, 30 \mathrm{mmol})$ and toluene $(100 \mathrm{~mL})$ was stirred at $80{ }^{\circ} \mathrm{C}$ for $10 \mathrm{~h}$. To this reaction mixture was added aqueous $\mathrm{NH}_{4} \mathrm{Cl}$, and the aqueous layer was extracted with $\mathrm{Et}_{2} \mathrm{O}$. The organic layer was washed with water and dried with $\mathrm{Na}_{2} \mathrm{SO}_{4}$ After evaporation of the solvents, the crude product was separated by WCC $\left(\mathrm{SiO}_{2}\right.$,

eluant: hexane/ $\left.\mathrm{CHCl}_{3} \quad 10: 1\right)$ to give 4,6-dibromo- $N, N$ '-bis(2-bromo-4-butylphenyl)benzene-1,3-diamine (11) as colorless oil (3.8 g, 55\%). To a THF solution $(50 \mathrm{~mL})$ of $\mathbf{1 1}(3.8 \mathrm{~g}, 5.5 \mathrm{mmol})$ at $-75{ }^{\circ} \mathrm{C}$ was added MeLi (1.0 M in $\mathrm{Et}_{2} \mathrm{O}, 17$ $\mathrm{mL}, 17 \mathrm{mmol})$. After the mixture was stirred for 30 minutes, MeI $(5.1 \mathrm{~mL}, 82 \mathrm{mmol})$ was added, and the mixture was warmed to r.t. and stirred for $4 \mathrm{~h}$. After addition of aqueous $\mathrm{NH}_{4} \mathrm{Cl}$, the aqueous layer was extracted with $\mathrm{CHCl}_{3}$, and the organic layer was dried with $\mathrm{Na}_{2} \mathrm{SO}_{4}$. After evaporation of the solvents, the crude product was subjected to column chromatography $\left(\mathrm{SiO}_{2}\right.$, eluant: hexane/ $\mathrm{CHCl}_{3}$ 10:1) to give 2 as colorless solids ( $0.99 \mathrm{~g}, 14 \%$ (2 steps)).

2: colorless solids, mp 110-112 ${ }^{\circ} \mathrm{C} .{ }^{1} \mathrm{H}$ NMR $\left(500 \mathrm{MHz}, \mathrm{CDCl}_{3}\right) \delta 0.90(\mathrm{t}, J=7.3 \mathrm{~Hz}, 6 \mathrm{H}), 1.32$ (sext, $J=7.4 \mathrm{~Hz}, 4 \mathrm{H}), 1.55$ (q, $J=7.6 \mathrm{~Hz}, 4 \mathrm{H}), 2.53(\mathrm{t}, J=7.7 \mathrm{~Hz}, 4 \mathrm{H}), 3.16(\mathrm{~s}, 6 \mathrm{H}), 6.70(\mathrm{~s}, 1 \mathrm{H})$, $6.82(\mathrm{~d}, J=8.1 \mathrm{~Hz}, 2 \mathrm{H}), 7.01(\mathrm{dd}, J=8.1,1.8 \mathrm{~Hz}, 2 \mathrm{H}), 7.38(\mathrm{~d}, J=1.8 \mathrm{~Hz}, 2 \mathrm{H}), 7.63(\mathrm{~s}, 1 \mathrm{H}) ;{ }^{13} \mathrm{C}$ NMR $\left(126 \mathrm{MHz}, \mathrm{CDCl}_{3}\right) \delta 13.92,22.22,33.34,34.59,41.23,112.67,116.69,120.62,124.22$, 128.19, 133.93, 138.44, 140.45, 145.62, 148.51; HRMS $\left(\mathrm{FAB}^{+}\right) \mathrm{m} / \mathrm{z}$ calcd for $\mathrm{C}_{28} \mathrm{H}_{32} \mathrm{~N}_{2}{ }^{79} \mathrm{Br}_{4}$ 711.9299, found: 711.9263. Anal. calcd for $\mathrm{C}_{28} \mathrm{H}_{32} \mathrm{Br}_{4} \mathrm{~N}_{2}$ : C, 46.96; H, 4.50; N, 3.91. Found. C, $47.00 ; \mathrm{H}, 4.51 ; \mathrm{N}, 3.64$.

\section{2,9-Dibutyl-5,12-dimethyl-7,14-dimesityl-5,7,12,14-tetrahydro-5,12-diaza-7,14-diborapentacen e (3)}

To a $\mathrm{Et}_{2} \mathrm{O}$ solution (1L) of $\mathbf{1}(0.71 \mathrm{~g}, 1.0 \mathrm{mmol})$ at $-75^{\circ} \mathrm{C}$ was added $t$-BuLi $(2.2 \mathrm{M}$ in pentane, 4.1 $\mathrm{mL}, 9.0 \mathrm{mmol}$ ), and the mixture was stirred for 30 minutes at $0{ }^{\circ} \mathrm{C}$. To this mixture was added $\mathrm{MesB}(\mathrm{OMe})_{2}(0.52 \mathrm{~mL}, 2.5 \mathrm{mmol})$. The resulting suspension was refluxed $1 \mathrm{~d}$ and filtered over 
Celite ${ }^{\circledR}$. After evaporation of the solvents, the crude product was recrystallized from $\mathrm{MeOH} / \mathrm{hexane} / \mathrm{AcOEt}$ to give 3 as red solids (0.36 g, 54\%).

3: red solids, mp 276-278 ${ }^{\circ} \mathrm{C} .{ }^{1} \mathrm{H}$ NMR $\left(500 \mathrm{MHz}, \mathrm{CDCl}_{3}\right) \delta 0.88(\mathrm{t}, J=7.3 \mathrm{~Hz}, 6 \mathrm{H}), 1.31$ (sext, $J=$ $7.3 \mathrm{~Hz}, 4 \mathrm{H}), 1.55$ (q, $J=7.8 \mathrm{~Hz}, 4 \mathrm{H}), 2.02(\mathrm{~s}, 12 \mathrm{H}), 2.46$ (s, 6H), $2.61(\mathrm{t}, J=7.6 \mathrm{~Hz}, 4 \mathrm{H}), 4.07$ (s, $6 \mathrm{H}), 7.00(\mathrm{~s}, 4 \mathrm{H}), 7.55-7.59(\mathrm{~m}, 4 \mathrm{H}), 7.64(\mathrm{~s}, 2 \mathrm{H}), 8.13(\mathrm{~s}, 2 \mathrm{H}) ;{ }^{13} \mathrm{C} \mathrm{NMR}\left(126 \mathrm{MHz}, \mathrm{CDCl}_{3}\right) \delta$ 13.96, 21.41, 22.31, 23.34, 34.20, 34.69, 35.37, 114.45, 121.61, 125.88, 126.86, 131.88, 132.76, 134.73, 136.17, 136.64, 139.15, 139.31, 140.28, 145.27; ${ }^{11} \mathrm{~B}$ NMR $\left(128 \mathrm{MHz}, \mathrm{CDCl}_{3}\right) \delta 54.26\left(h_{1 / 2}\right.$ $=1900 \mathrm{~Hz}$ ); HRMS $\left(\mathrm{FAB}^{+}\right) \mathrm{m} / \mathrm{z}$ calcd for $\mathrm{C}_{46} \mathrm{H}_{54} \mathrm{~N}_{2}{ }^{10} \mathrm{~B}^{11} \mathrm{~B}$ 655.4509, found: 655.4454; UV-vis (cyclohexane) $\lambda / \mathrm{nm}(\log \varepsilon): 523$ (4.23), 490 (3.97), 321 (4.31).

\section{2,10-Dibutyl-5,7-dimethyl-12,14-dimesityl-5,7,12,14-tetrahydro-5,7-diaza-12,14-diborapentace ne (4)}

To a $\mathrm{Et}_{2} \mathrm{O}$ solution $(300 \mathrm{~mL})$ of $2(200 \mathrm{mg}, 0.28 \mathrm{mmol})$ at $-75^{\circ} \mathrm{C}$ was added $t$-BuLi $(2.2 \mathrm{M}$ in pentane, $1.1 \mathrm{~mL}, 2.4 \mathrm{mmol}$ ), and the mixture was stirred for 30 minutes at $0{ }^{\circ} \mathrm{C}$. To this mixture was added $\mathrm{MesB}(\mathrm{OMe})_{2}(0.14 \mathrm{~mL}, 0.68 \mathrm{mmol})$. The resultant suspension was refluxed $1 \mathrm{~d}$ and filtered over Celite ${ }^{\circledR}$ pad, and the solvents were removed under reduced pressure. The resulting solids were extracted with $\mathrm{CHCl}_{3}$. The solution was filtered and evaporated. The crude product was recrystallized from $\mathrm{CHCl}_{3} / \mathrm{MeOH}$ to give 4 as yellow solids (97 mg, 54\%).

4: yellow solids, mp 311-313 ${ }^{\circ} \mathrm{C} .{ }^{1} \mathrm{H}$ NMR $\left(500 \mathrm{MHz}, \mathrm{CDCl}_{3}\right) \delta 0.87$ (t, $\left.J=7.3 \mathrm{~Hz}, 6 \mathrm{H}\right), 1.31$ (sext, $J=7.4 \mathrm{~Hz}, 4 \mathrm{H}), 1.54$ (q, $J=7.7 \mathrm{~Hz}, 4 \mathrm{H}), 1.84$ (s, 12H), 2.35 (s, 6H), 2.61 ( $t, \mathrm{~J}=7.8 \mathrm{~Hz}, 4 \mathrm{H}), 4.10$ (s, 6H), $6.76(\mathrm{~s}, 4 \mathrm{H}), 7.34(\mathrm{~s}, 1 \mathrm{H}), 7.54-7.59(\mathrm{~m}, 4 \mathrm{H}), 7.70$ (d, J = 1.7 Hz, 2H), $7.99(\mathrm{~s}, 1 \mathrm{H}) ;{ }^{13} \mathrm{C} \mathrm{NMR}$ $\left(100 \mathrm{MHz}, \mathrm{CDCl}_{3}\right) \delta 13.97,21.22,22.40,23.12,34.29,34.68,35.67,96.50,114.74,119.46,120.76$, $126.28,133.82,134.26,135.85,136.87,138.86,139.44,145.53,150.10,151.61 ;{ }^{11} \mathrm{~B}$ NMR (128

$\mathrm{MHz}, \mathrm{CDCl} 3) \delta 53.0\left(h_{1 / 2}=1900 \mathrm{~Hz}\right)$; HRMS $\left(\mathrm{FAB}^{+}\right) \mathrm{m} / \mathrm{z}$ calcd for $\mathrm{C}_{46} \mathrm{H}_{54} \mathrm{~N}_{2}{ }^{10} \mathrm{~B}^{11} \mathrm{~B} 655.4509$, found: 655.4485; UV-vis (cyclohexane) $\lambda_{\max } / \mathrm{nm}(\log \varepsilon): 415$ (3.92), 389 (3.99), 323 (4.67), 272 (4.63).

\section{2-Bromo-4-dodecylaniline}

A mixture of 4-dodecylaniline (45 g, $0.17 \mathrm{~mol}), \mathrm{NaVO}_{3}(2.1 \mathrm{~g}, 17 \mathrm{mmol}), \mathrm{KBr}(21 \mathrm{~g}, 0.18 \mathrm{~mol})$, $\mathrm{NaBO}_{3} \cdot 4 \mathrm{H}_{2} \mathrm{O}(27 \mathrm{~g}, 0.17 \mathrm{~mol})$ and $\mathrm{AcOH}(200 \mathrm{~mL})$ was stirred at r.t. for $10 \mathrm{~h}$ under air. The mixture was neutralized with aqueous $\mathrm{Na}_{2} \mathrm{CO}_{3}$, and the aqueous layer was extracted with $\mathrm{Et}_{2} \mathrm{O}$. The organic layer was washed by aqueous $\mathrm{Na}_{2} \mathrm{SO}_{3}$ and $\mathrm{H}_{2} \mathrm{O}$ and dried over $\mathrm{Na}_{2} \mathrm{SO}_{4}$. Crude product was subjected to column chromatography $\left(\mathrm{SiO}_{2}\right.$, eluent: $\left.\mathrm{CHCl}_{3}\right)$ to give the title compound as pale red solids $(34 \mathrm{~g}$, $59 \%)$.

Pale red solids, mp 38-40 ${ }^{\circ} \mathrm{C} .{ }^{1} \mathrm{H}$ NMR $\left(500 \mathrm{MHz}, \mathrm{CDCl}_{3}\right) \delta 0.87(\mathrm{t}, J=7.0 \mathrm{~Hz}, 3 \mathrm{H}), 1.24-1.27(\mathrm{~m}$, $18 \mathrm{H}), 1.52$ (quint, $J=7.5 \mathrm{~Hz}, 2 \mathrm{H}), 2.45(\mathrm{t}, J=7.7 \mathrm{~Hz}, 2 \mathrm{H}), 6.68(\mathrm{~d}, J=8.1 \mathrm{~Hz}, 1 \mathrm{H}), 6.90$ (dd, $J=$ 
8.1, 1.8 Hz, 1H), $7.22(\mathrm{~d}, J=1.8 \mathrm{~Hz}, 1 \mathrm{H}) ;{ }^{13} \mathrm{C} \mathrm{NMR}(126 \mathrm{MHz}, \mathrm{CDCl} 3) \delta 14.11,22.67,29.15,29.34$, 29.47, 29.57, 29.62, 31.59, 31.90, 34.66, 109.27, 115.70, 128.31, 132.05, 134.34, 141.63 (Two signals of the dodecyl groups could not be observed due to overlapping of the signals.); HRMS $\left(\mathrm{FAB}^{+}\right) \mathrm{m} / \mathrm{z}$ calcd for $\mathrm{C}_{18} \mathrm{H}_{30} \mathrm{~N}^{79} \mathrm{Br} 339.1562$, found: 339.1593 .

\section{(2-Bromo-4-dodecylphenyl)(2,5-dibromo-4-iodophenyl)methylamine (5)}

A mixture of 2-bromo-4-dodecylaniline (10 g, $29 \mathrm{mmol})$, 1,4-dibromo-2,5-diiodobenzene (14 g, 29 mmol), Pd(dba) $)_{2}(0.83 \mathrm{~g}, 0.14 \mathrm{mmol}), \operatorname{DPPF}(0.83 \mathrm{~g}, 0.14 \mathrm{mmol}), t-\mathrm{BuONa}(4.2 \mathrm{~g}, 44 \mathrm{mmol})$ and toluene $(50 \mathrm{~mL})$ was stirred at $100{ }^{\circ} \mathrm{C}$ for $1 \mathrm{~d}$. The reaction was quenched by the addition of aqueous $\mathrm{NH}_{4} \mathrm{Cl}$, and the aqueous layer was extracted with $\mathrm{Et}_{2} \mathrm{O}$. The organic layer was dried with $\mathrm{Na}_{2} \mathrm{SO}_{4}$, and the solvents were evaporated. The crude product was dissolved in THF $(50 \mathrm{~mL})$, and to this mixture was added MeI (2.8 mL, $45 \mathrm{mmol}), \mathrm{KOH}(17 \mathrm{~g}, 0.30 \mathrm{~mol}), \mathrm{Bu}_{4} \mathrm{NI}(2.2 \mathrm{~g}, 6.0 \mathrm{mmol})$. After stirring at $50{ }^{\circ} \mathrm{C}$, the reaction was quenched by the addition of aqueous $\mathrm{NH}_{4} \mathrm{Cl}$, and the aqueous layer was extracted with $\mathrm{Et}_{2} \mathrm{O}$. The organic layer was washed with water and dried with $\mathrm{Na}_{2} \mathrm{SO}_{4}$, and the solvents were evaporated. The crude product was separated by $\mathrm{WCC}\left(\mathrm{SiO}_{2}\right.$, eluant: hexane), and recrystallization from hexane gave 5 as pale brown oil (12 g, 55\%).

5: pale brown oil, ${ }^{1} \mathrm{H}$ NMR $\left(500 \mathrm{MHz}, \mathrm{CDCl}_{3}\right) \delta 0.86(\mathrm{t}, J=6.9 \mathrm{~Hz}, 3 \mathrm{H}), 1.24-1.29(\mathrm{~m}, 18 \mathrm{H}), 1.58$ (q, $J=8.1 \mathrm{~Hz}, 2 \mathrm{H}), 2.53$ (t, $J=7.8 \mathrm{~Hz}, 2 \mathrm{H}), 3.16(\mathrm{~s}, 3 \mathrm{H}), 6.80$ (d, $J=8.1 \mathrm{~Hz}, 1 \mathrm{H}), 7.03$ (dd, $J=8.1$, $1.9 \mathrm{~Hz}, 1 \mathrm{H}), 7.25(\mathrm{~s}, 1 \mathrm{H}), 7.40(\mathrm{~d}, J=1.9 \mathrm{~Hz}, 1 \mathrm{H}), 7.91(\mathrm{~s}, 1 \mathrm{H}) ;{ }^{13} \mathrm{C} \mathrm{NMR}\left(126 \mathrm{MHz}, \mathrm{CDCl}_{3}\right) \delta$ $14.14,22.68,29.17,29.34,29.42,29.54,29.62,31.18,31.57,31.90,34.63,34.94,41.38,92.62$, 117.87, 121.07, 124.71, 125.82, 126.30, 126.91, 133.99, 141.25, 143.92, 144.90, 149.71; HRMS $\left(\mathrm{FAB}^{+}\right) \mathrm{m} / \mathrm{z}$ calcd for $\mathrm{C}_{25} \mathrm{H}_{33} \mathrm{~N}^{79} \mathrm{Br}_{3} \mathrm{I} 710.9208$, found: 710.9191

\section{2,5-Dibromo- $N$-(2-bromo-4-dodecylphenyl)- $N$-methylbenzene-1,4-diamine (6)}

A mixture of 5 ( $8.9 \mathrm{~g}, 12 \mathrm{mmol})$, benzophenone imine $(2.4 \mathrm{~mL}, 14 \mathrm{mmol}), \operatorname{Pd}(\mathrm{dba})_{2}(0.40 \mathrm{~g}, 0.69$ mmol), DPPF (0.40 g, $0.70 \mathrm{mmol}), t$-BuONa $(1.9 \mathrm{~g}, 20 \mathrm{mmol})$ and toluene $(50 \mathrm{~mL})$ was stirred at $100{ }^{\circ} \mathrm{C}$ for $1 \mathrm{~d}$. The reaction was quenched by the addition of aqueous $\mathrm{NH}_{4} \mathrm{Cl}$, and the aqueous layer was extracted with $\mathrm{Et}_{2} \mathrm{O}$. The organic layer was dried with $\mathrm{Na}_{2} \mathrm{SO}_{4}$, and the solvents were evaporated. The crude product was separated by column chromatography $\left(\mathrm{SiO}_{2}\right.$, eluant: $\left.\mathrm{CHCl}_{3}\right)$ to give $N$-benzhydrylidene-2,5-dibromo- $N$ '-(2-bromo-4-dodecylphenyl)- $N$ '-methylbenzene-1,4-diamine (12) as red oil $(8.8 \mathrm{~g}, 98 \%) .12$ was dissolved in $\mathrm{MeOH}(20 \mathrm{~mL})$ and THF $(5 \mathrm{~mL})$. To this mixture were added $\mathrm{NH}_{2} \mathrm{OH} \cdot \mathrm{HCl}(5.3 \mathrm{~g}, 77 \mathrm{mmol})$ and $\mathrm{AcONa}(11 \mathrm{~g}, 0.13 \mathrm{~mol})$, and the mixture was stirred at r.t. for $1 \mathrm{~d}$. The reaction was quenched by the addition of aqueous $\mathrm{NaOH}(0.1 \mathrm{M})$, and the aqueous layer was extracted with $\mathrm{CH}_{2} \mathrm{Cl}_{2}$. The organic layer was dried with $\mathrm{Na}_{2} \mathrm{SO}_{4}$, and the solvents were evaporated. The crude product was separated by column chromatography $\left(\mathrm{SiO}_{2}\right.$, eluant: hexane/AcOEt 6:1) to give 6 as orange oil (4.5 g, 63\%). 
6: orange oil, ${ }^{1} \mathrm{H}$ NMR $\left(400 \mathrm{MHz}, \mathrm{CDCl}_{3}\right) \delta 0.88(\mathrm{t}, J=6.8 \mathrm{~Hz}, 3 \mathrm{H}), 1.25$ (brs, $\left.18 \mathrm{H}\right), 1.57$ (q, $J=$ $7.4 \mathrm{~Hz}, 2 \mathrm{H}), 2.52(\mathrm{t}, J=7.8 \mathrm{~Hz}, 2 \mathrm{H}), 3.11$ (s, 3H), 4.01 (brs, 2H), 6.90 (d, J = 8.0 Hz, 1H), 6.99 (s, $1 \mathrm{H}), 7.00(\mathrm{~s}, 1 \mathrm{H}), 7.03(\mathrm{dd}, J=8.0,1.5 \mathrm{~Hz}, 1 \mathrm{H}), 7.35(\mathrm{~d}, J=1.5 \mathrm{~Hz}, 1 \mathrm{H}) ;{ }^{13} \mathrm{C}$ NMR $(126 \mathrm{MHz}$, $\left.\mathrm{CDCl}_{3}\right) \delta 14.12,23.00,29.20,29.33,29.42,29.53,29.61,31.22,31.88,34.82,41.74,107.95,119.24$, $119.57,120.05,122.56,127.78,127.94,134.04,139.42$, 140.81, 141.29, 145.96 (Two signals of the dedecyl groups could not be observed due to the overlapping of the signals.); HRMS (FAB ${ }^{+}$) $\mathrm{m} / \mathrm{z}$ calcd for $\mathrm{C}_{25} \mathrm{H}_{35} \mathrm{~N}_{2}{ }^{79} \mathrm{Br}_{3} 600.0350$, found: 600.0325 .

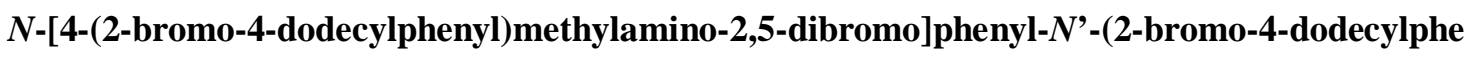
nyl)-2,5-dibromo- $N, N$ '-dimethylbenzene-1,4-diamine (7)

A mixture of $5(5.4 \mathrm{~g}, 7.6 \mathrm{mmol}), 6(4.5 \mathrm{~g}, 7.5 \mathrm{mmol}), \operatorname{Pd}(\mathrm{dba})_{2}(0.22 \mathrm{~g}, 0.38 \mathrm{mmol})$, DPPF $(0.21 \mathrm{~g}$, $0.37 \mathrm{mmol}), t$-BuONa $(1.1 \mathrm{~g}, 11 \mathrm{mmol})$ and toluene $(50 \mathrm{~mL})$ was stirred at $100{ }^{\circ} \mathrm{C}$ for $31 \mathrm{~h}$. To this reaction mixture was added aqueous $\mathrm{NH}_{4} \mathrm{Cl}$, and the aqueous layer was extracted with hexane. The organic layer was dried with $\mathrm{Na}_{2} \mathrm{SO}_{4}$. After evaporation of the solvents, the crude product was separated by WCC $\left(\mathrm{SiO}_{2}\right.$, eluant: hexane/ $\left.\mathrm{CHCl}_{3} 4: 1\right)$ to give $\mathbf{1 3}$ as brown oil (5.8 g, 59\%). To a THF solution $(50 \mathrm{~mL})$ of $\mathbf{1 3}(5.8 \mathrm{~g}, 4.4 \mathrm{mmol})$ at $-75^{\circ} \mathrm{C}$ was added $\mathrm{MeLi}\left(1.0 \mathrm{M}\right.$ in $\mathrm{Et}_{2} \mathrm{O}, 15 \mathrm{~mL}, 15$ $\mathrm{mmol})$. After the mixture was stirred for 30 minutes, MeI $(3.0 \mathrm{~mL}, 48 \mathrm{mmol})$ was added, and the mixture was warmed to r.t. and stirred for $3 \mathrm{~h}$. After addition of aqueous $\mathrm{NH}_{4} \mathrm{Cl}$, the aqueous layer wasexttracted with $\mathrm{Et}_{2} \mathrm{O}$, and the organic layer was washed with aqueous $\mathrm{NaCl}$ and dried over $\mathrm{Na}_{2} \mathrm{SO}_{4}$. After evaporation of the solvents, the crude product was subjected to column chromatography $\left(\mathrm{SiO}_{2}\right.$, eluant: $\left.\mathrm{CHCl}_{3}\right)$ and recrystallized from $\mathrm{C}_{6} \mathrm{H}_{6}$ to give $\mathbf{7}$ as colorless solids (3.9 g, $43 \%$ (2 steps)).

7: colorless solids, mp 180-182 ${ }^{\circ} \mathrm{C} .{ }^{1} \mathrm{H}$ NMR $\left(500 \mathrm{MHz}, \mathrm{CDCl}_{3}\right) \delta 0.86(\mathrm{t}, J=6.9 \mathrm{~Hz}, 6 \mathrm{H}), 1.24$ (brs, 26H), 1.29 (brs, 10H), 1.57 (q, $J=7.1 \mathrm{~Hz}, 4 \mathrm{H}), 2.52$ (t, $J=7.8 \mathrm{~Hz}, 4 \mathrm{H}), 3.14$ (s, 3H), 3.16 (s, 6H), $6.86(\mathrm{~d}, J=8.1 \mathrm{~Hz}, 2 \mathrm{H}), 7.03$ (dd, $J=8.1,1.8 \mathrm{~Hz}, 2 \mathrm{H}), 7.13(\mathrm{~s}, 2 \mathrm{H}), 7.18(\mathrm{~s}, 2 \mathrm{H}), 7.38$ (d, $J=1.8 \mathrm{~Hz}$, $2 \mathrm{H}) ;{ }^{13} \mathrm{C} \mathrm{NMR}\left(126 \mathrm{MHz}, \mathrm{CDCl}_{3}\right) \delta 14.13,22.68 .29 .22,29.34,29.43,29.54,29.63,29.65,31.22$, 31.90, 34.92, 41.52 (Two signals of the dodecyl groups are overlapped.), 118.76, 119.11, 120.35, 123.82, 128.19, 128.71 (Two signals in the aromatic region are overlapped.), 134.04, 140.41, 144.17, $145.49,145.53$ (One signal corresponding to a methylene carbon of the dodecyl groups could not be observed due to overlapping of the signals.); HRMS (FAB ${ }^{+}$m/z calcd for $\mathrm{C}_{51} \mathrm{H}_{69} \mathrm{~N}_{3}{ }^{79} \mathrm{Br}_{3}{ }^{81} \mathrm{Br}_{3}$ 1203.0530, found: 1203.0500. Anal. calcd for $\mathrm{C}_{51} \mathrm{H}_{69} \mathrm{Br}_{6} \mathrm{~N}_{3}$ : C, 50.90; H, 5.78; N, 3.49. Found: $\mathrm{C}$, $50.84 ; \mathrm{H}, 5.84 ; \mathrm{N}, 3.27$. 
<smiles>CCCCc1ccc(N(C)c2cc(Br)c(Nc3cc(Br)c(N(C)c4ccc(CC(C)C)cc4Br)cc3Br)cc2Br)c(Br)c1</smiles>

2,12-Didodecyl-5,9,16-trimethyl-7,14,18-trimesityl-5,7,9,14,16,18-hexahydro-5,9,16-triaza-7,14, 18-triboraheptacene (8)

To a $\mathrm{Et}_{2} \mathrm{O}$ solution $(500 \mathrm{~mL})$ of $7(0.50 \mathrm{~g}, 0.42 \mathrm{mmol})$ at $-75^{\circ} \mathrm{C}$ was added $t-\mathrm{BuLi}(2.2 \mathrm{M}$ in pentane, $2.5 \mathrm{~mL}, 5.5 \mathrm{mmol}$ ), and the mixture was stirred for 10 minutes then at $0{ }^{\circ} \mathrm{C}$ for 10 minutes. To the mixture at $-75{ }^{\circ} \mathrm{C}$ was added $\mathrm{MesB}(\mathrm{OMe})_{2}(0.35 \mathrm{~mL}, 1.7 \mathrm{mmol})$. The resulting mixture was refluxed for $12 \mathrm{~h}$ and filtered by Celite ${ }^{\circledR}$ pad, and the solvents were evaporated. The crude product was subjected to column chromatography $\left(\mathrm{Al}_{2} \mathrm{O}_{3}\right.$, eluant: $\mathrm{C}_{6} \mathrm{H}_{6} /$ hexane 1:4) to give 8 as purple solids $(0.21 \mathrm{~g}, 46 \%)$

8: purple solids, mp $210-212{ }^{\circ} \mathrm{C} .{ }^{1} \mathrm{H}$ NMR $\left(500 \mathrm{MHz}, \mathrm{CDCl}_{3}\right) \delta 0.86(\mathrm{t}, J=7.0 \mathrm{~Hz}, 6 \mathrm{H}), 1.21-1.28$ (m, 40H), 2.00 (s, 12H), 2.08 (s, 6H), 2.43 (s, 6H), 2.49 (s, 3H), 2.60 (t, J= 7.6 Hz, 4H), $3.80(\mathrm{~s}, 3 \mathrm{H})$, $3.95(\mathrm{~s}, 6 \mathrm{H}), 6.95(\mathrm{~s}, 4 \mathrm{H}), 7.04(\mathrm{~s}, 2 \mathrm{H}), 7.55-7.60(\mathrm{~m}, 4 \mathrm{H}), 7.67(\mathrm{~d}, J=1.5 \mathrm{~Hz}, 2 \mathrm{H}), 8.06(\mathrm{~s}, 2 \mathrm{H})$, 8.19 (s, 2H); ${ }^{13} \mathrm{C}$ NMR $\left(126 \mathrm{MHz}, \mathrm{CDCl}_{3}\right) \delta 14.14,21.39,21.47,22.69,23.30,23.47,29.22,29.36$, $29.53,29.65,31.92,32.07,34.93,35.34,35.41,114.45,121.41,121.93,125.81,126.86,127.04$, $130.70,132.67,132.75,134.82,136.19,136.39,136.80,138.72,139.20,139.26,139.68,140.04$, 140.19, 145.33 (Four methylene signals of the dodecyl groups are overlapped.); HRMS (FAB $\left.{ }^{+}\right) \mathrm{m} / \mathrm{z}$ calcd for $\mathrm{C}_{78} \mathrm{H}_{102} \mathrm{~N}_{3}{ }^{10} \mathrm{~B}_{2}{ }^{11} \mathrm{~B} 1111.8426$, found: 1111.8400; UV-vis (cyclohexane) $\lambda_{\max } / \mathrm{nm}(\log \varepsilon): 608$ (4.28), 565 (3.95), 438 (3.36), 318 (4.48).

\section{References for Supporting Information}

1) Gilman, H.; Zuech, E. A. J. Org. Chem. 1959, 24, 1394.

2) Hart, H.; Harada, K.; Du, C.-J. F. J. Org. Chem. 1985, 50, 3104.

3) Bandgar, B. P.; Nigal, M. N. J. Synth. Commun. 1998, 28, 3225. 
$1{ }^{1} \mathrm{H}$ NMR

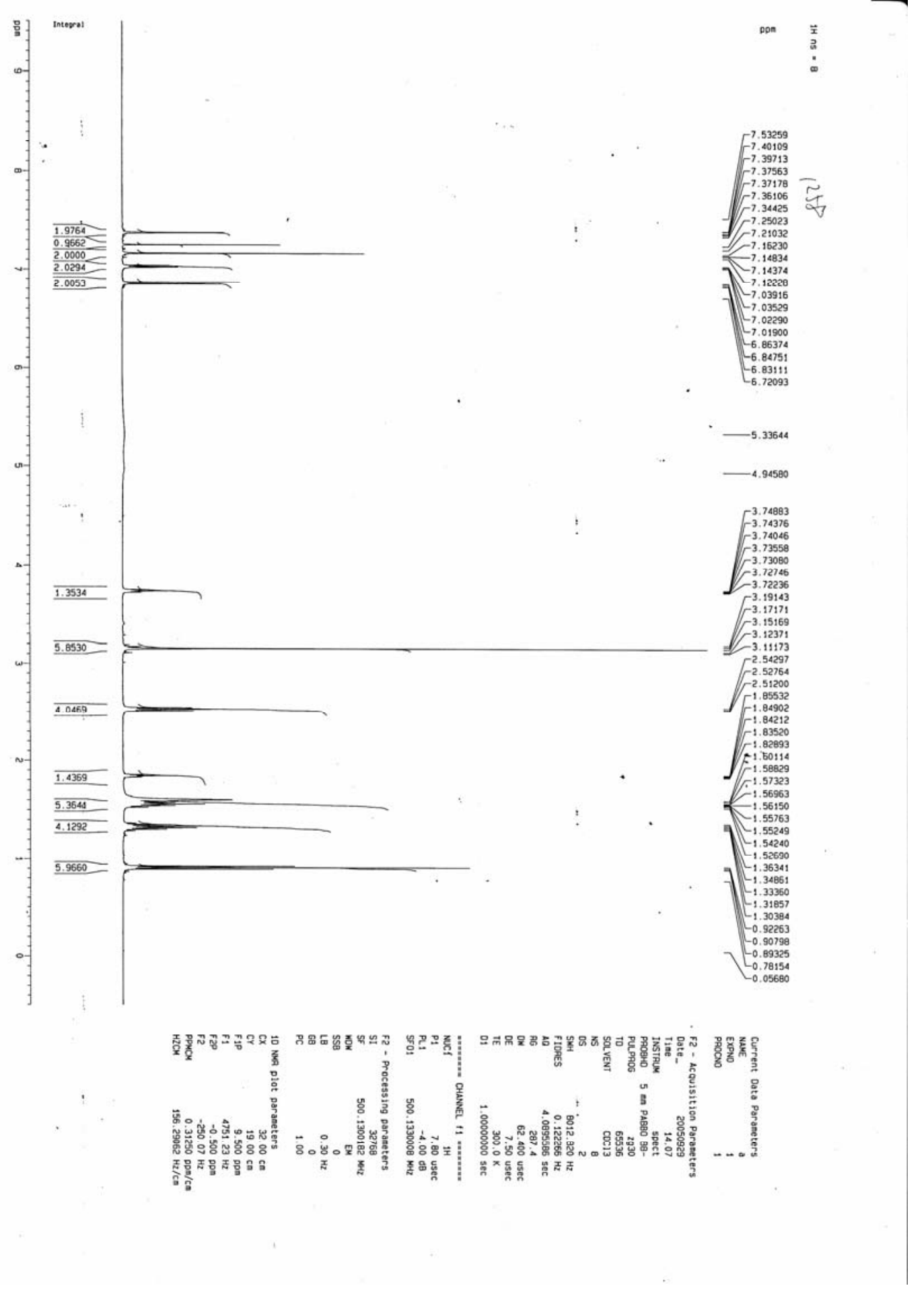


$\mathbf{1}^{13} \mathrm{C}$ NMR

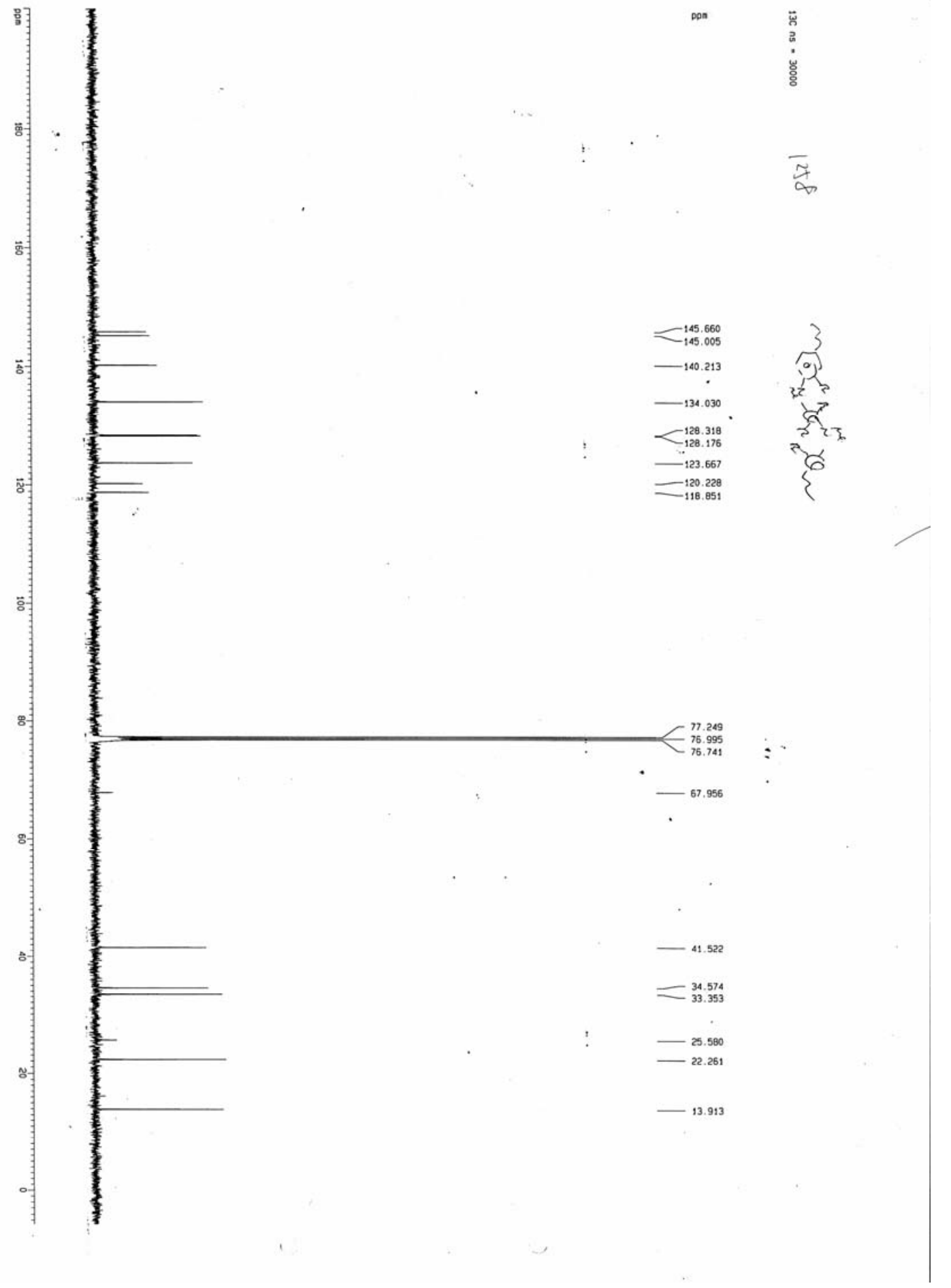




\section{$2{ }^{1} \mathrm{H}$ NMR}

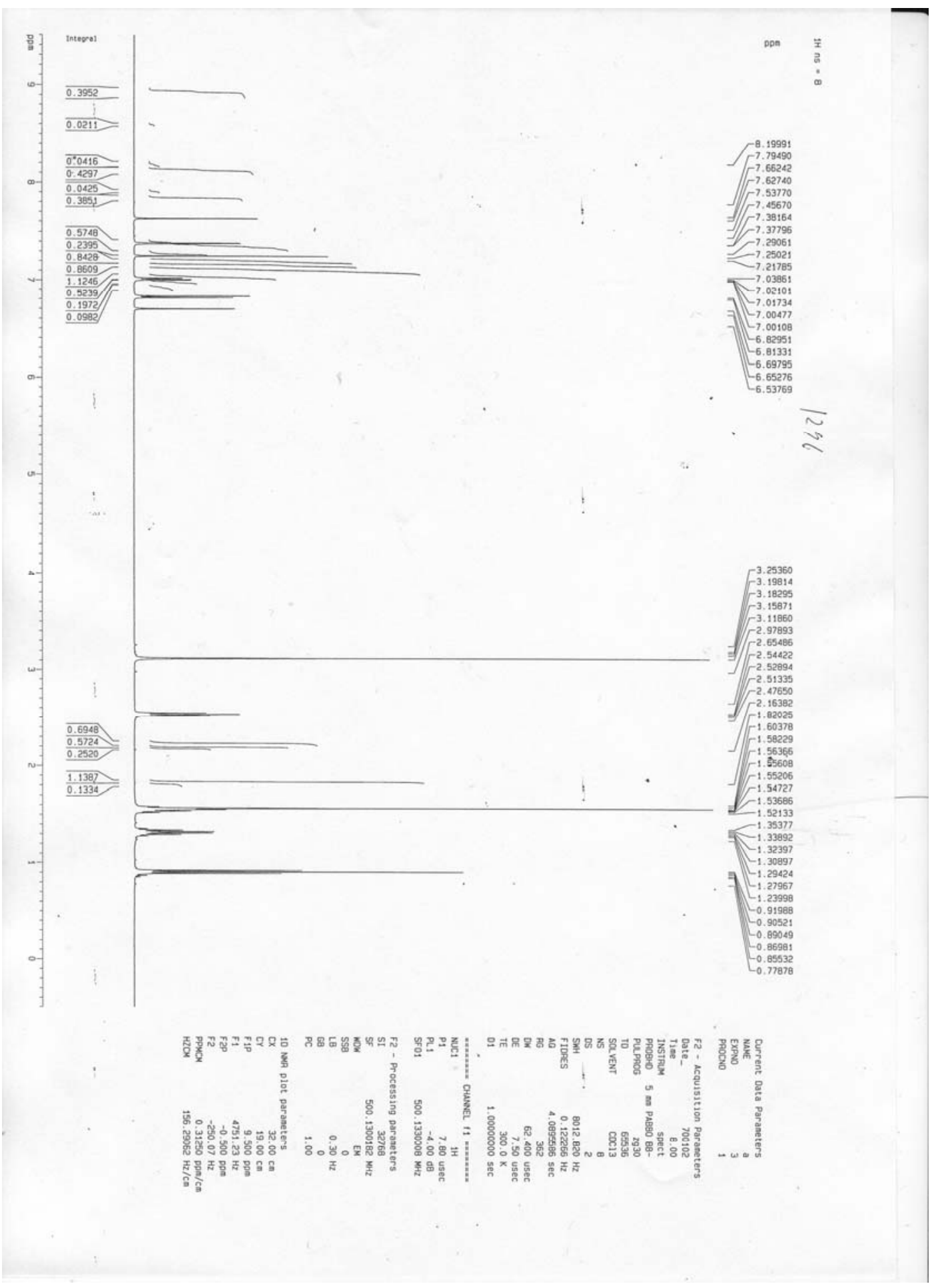




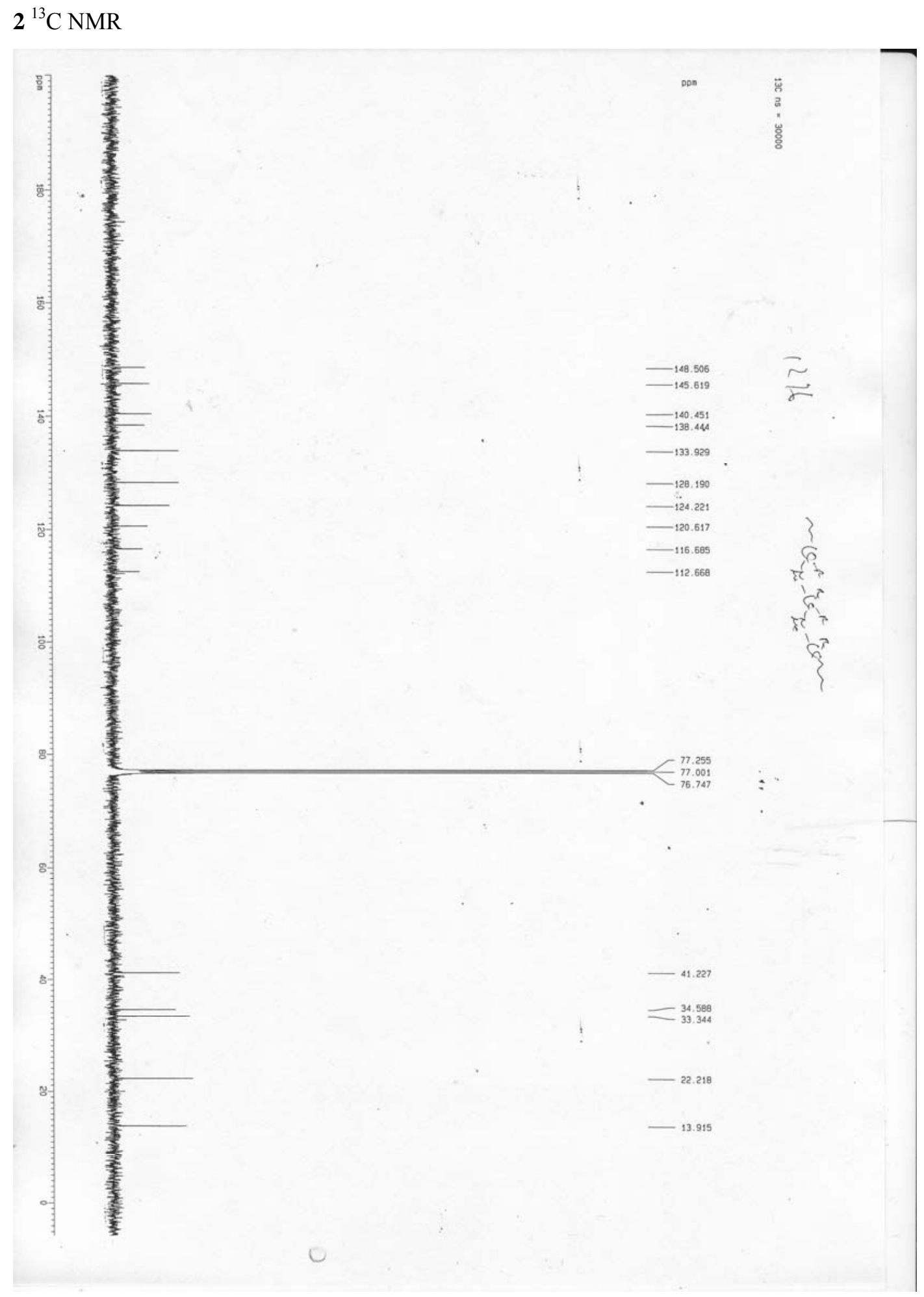




\section{$3{ }^{1} \mathrm{H}$ NMR}

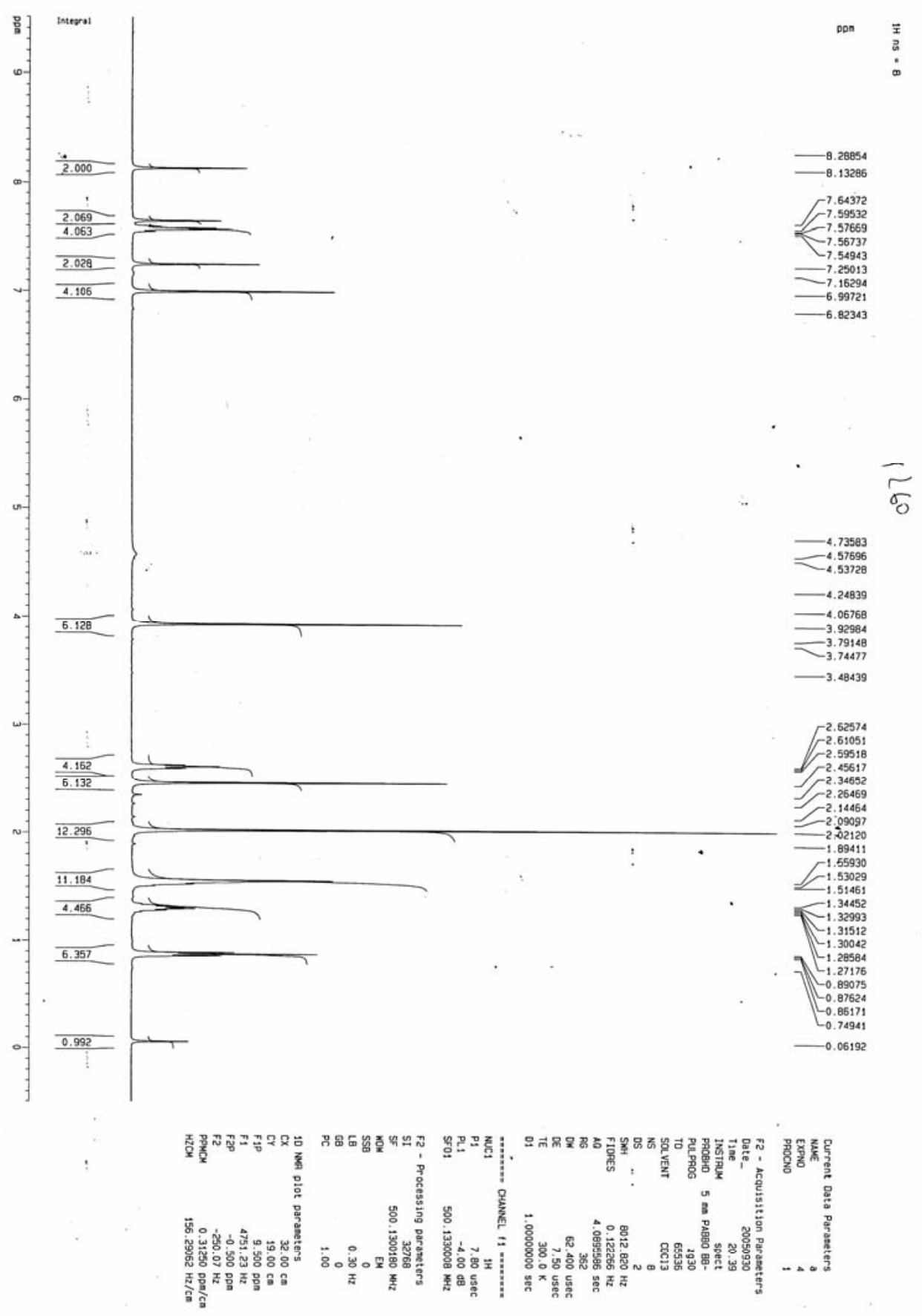




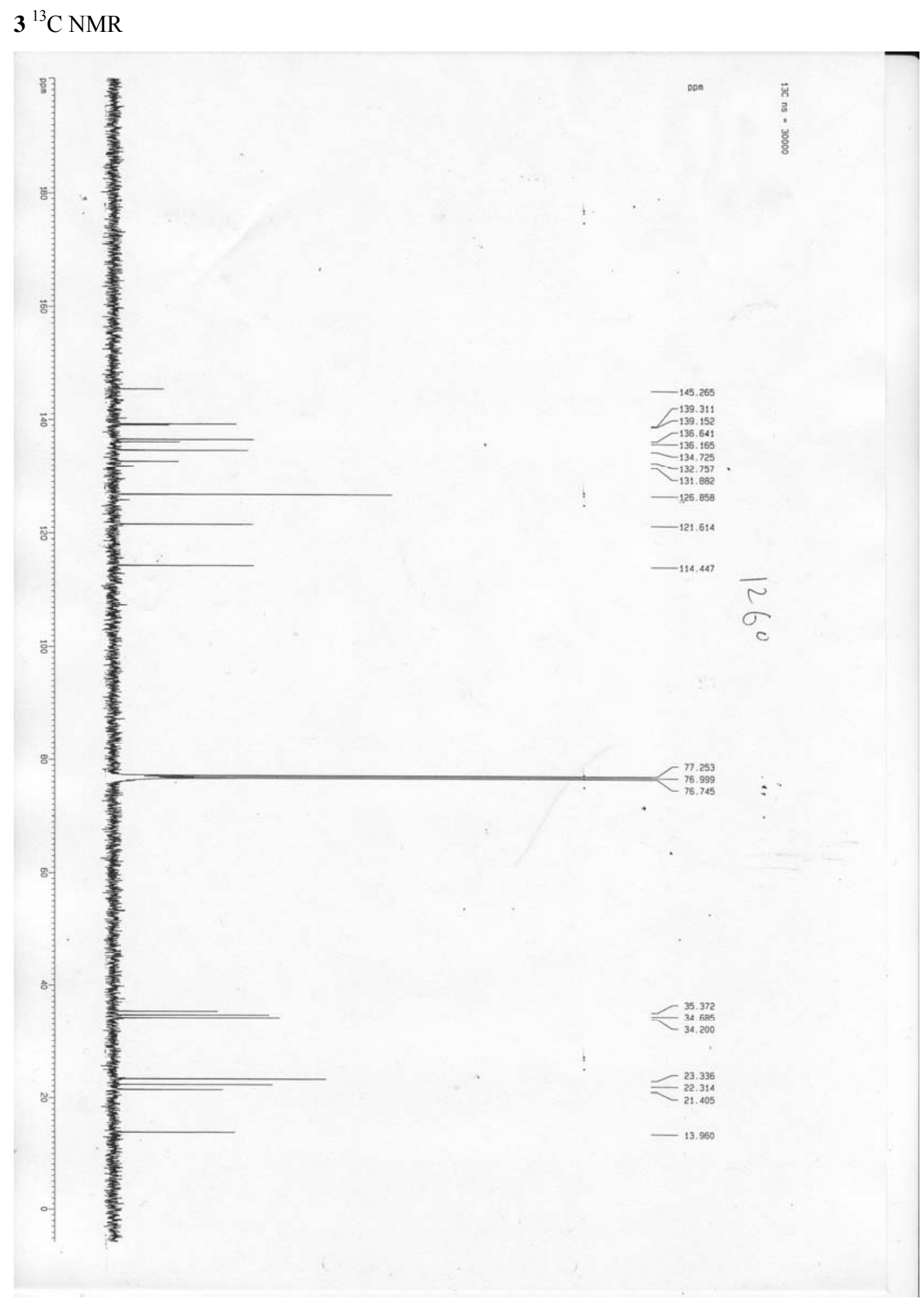




\section{$4{ }^{1} \mathrm{H}$ NMR}

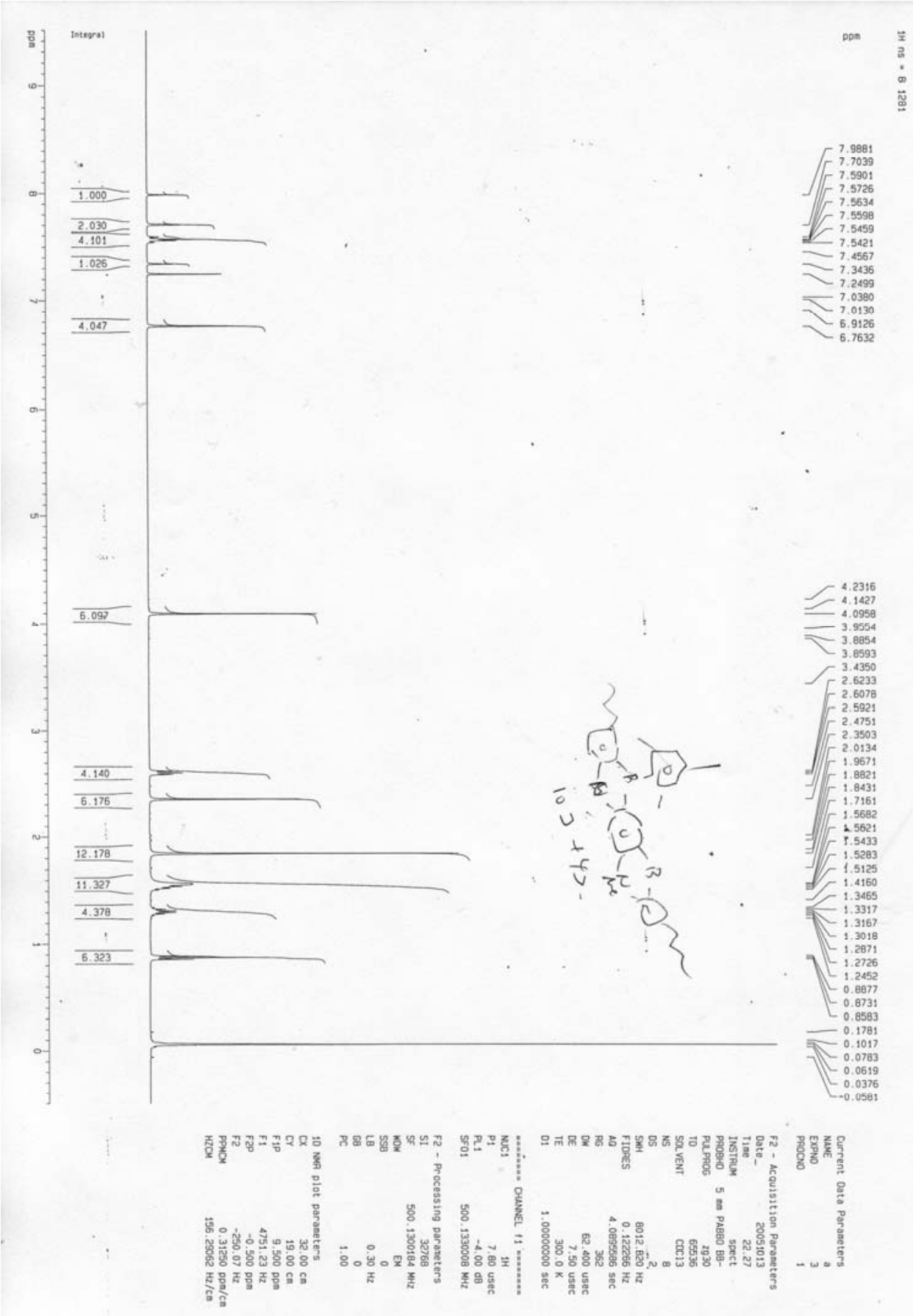


$4{ }^{13} \mathrm{C}$ NMR

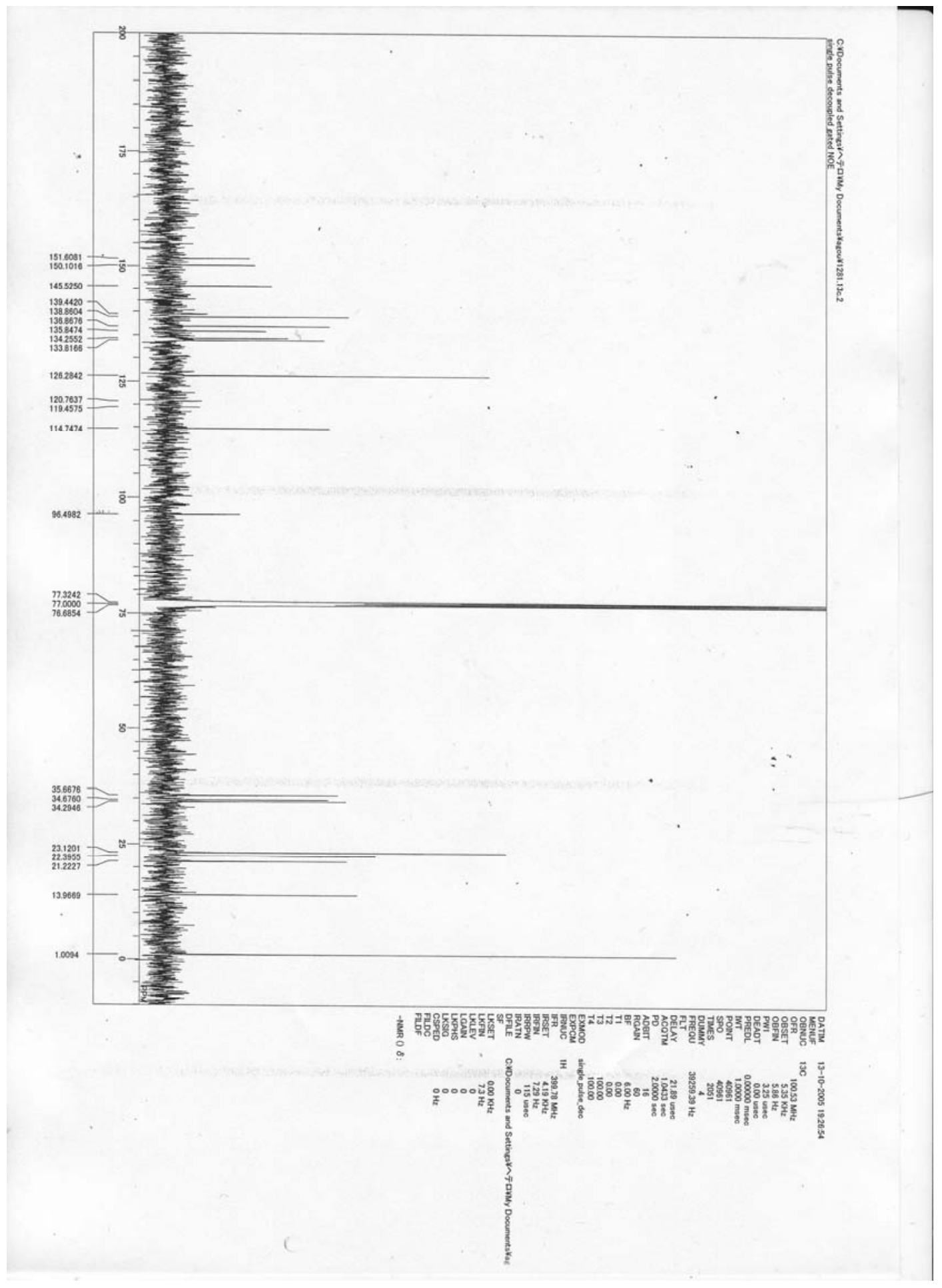


$5{ }^{1} \mathrm{H}$ NMR

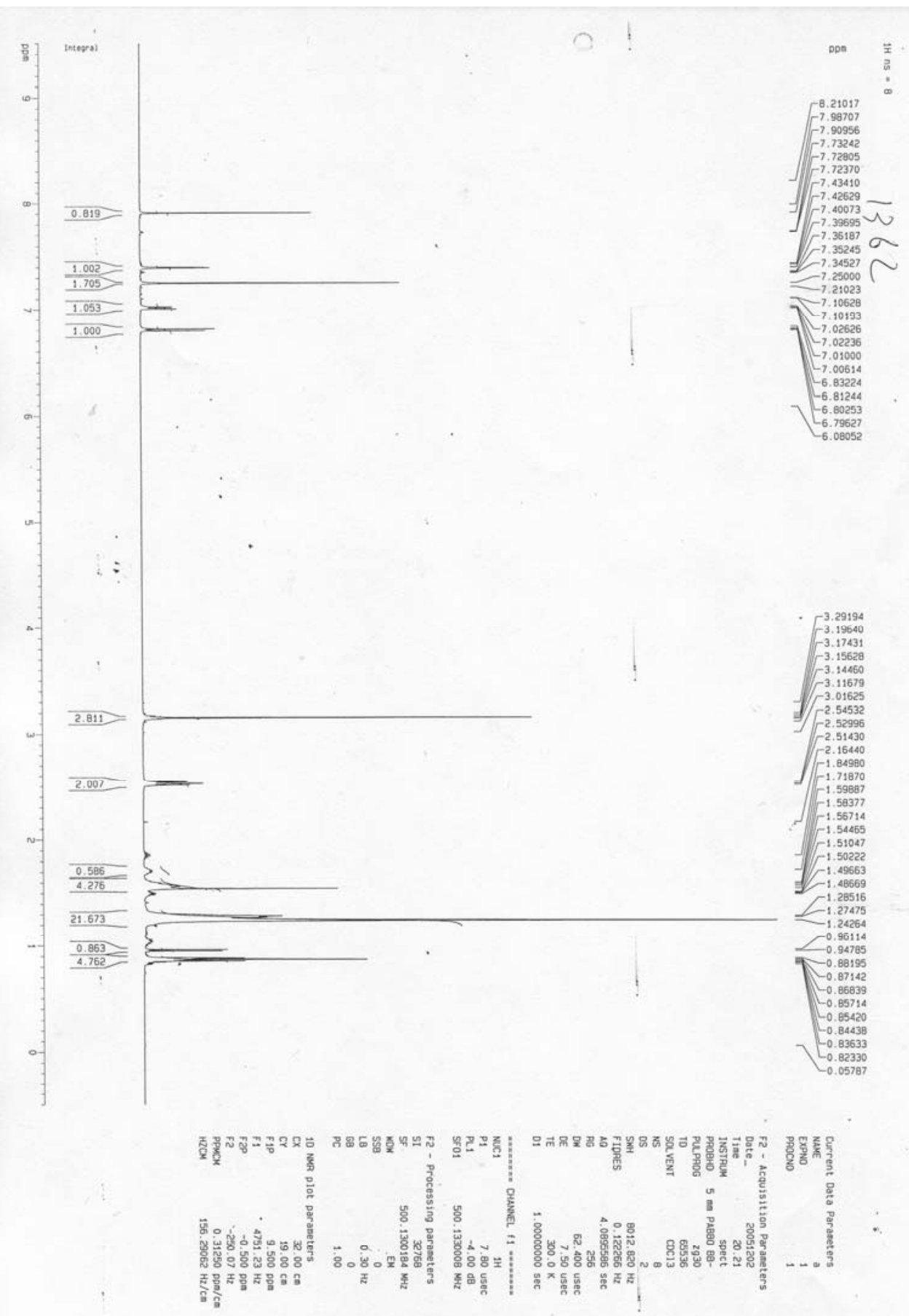


$5{ }^{13} \mathrm{C}$ NMR

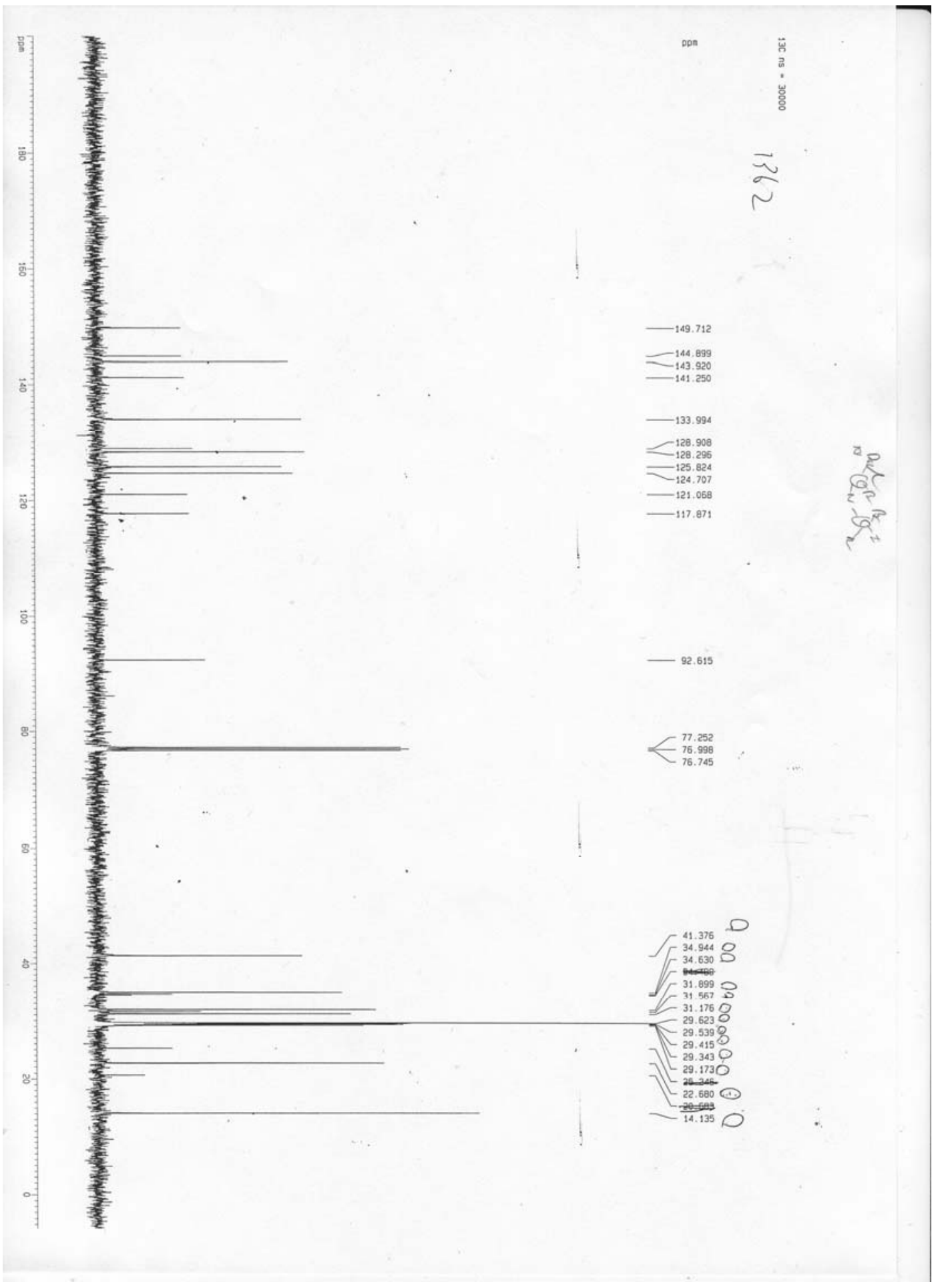


$6{ }^{1} \mathrm{H}$ NMR

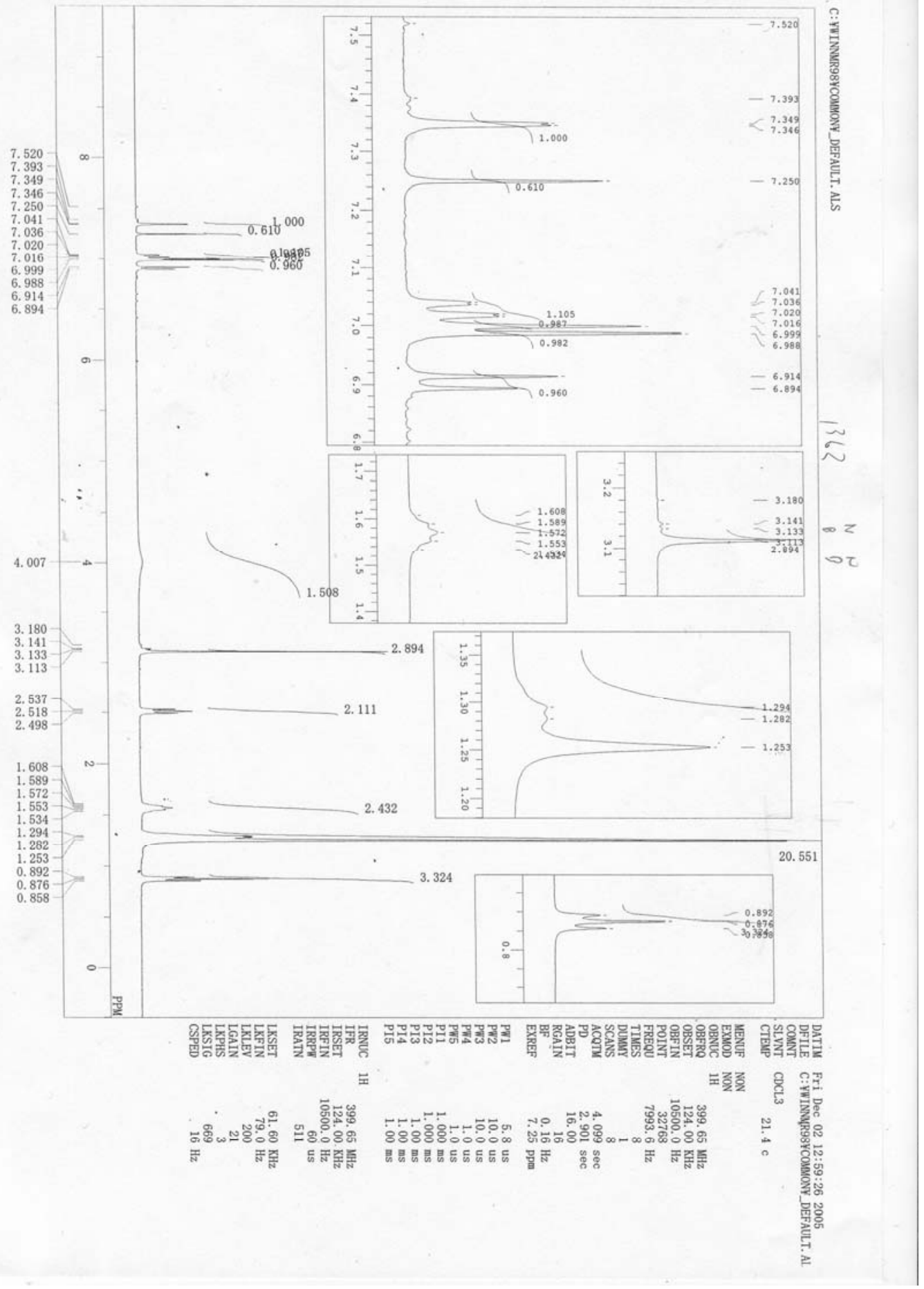




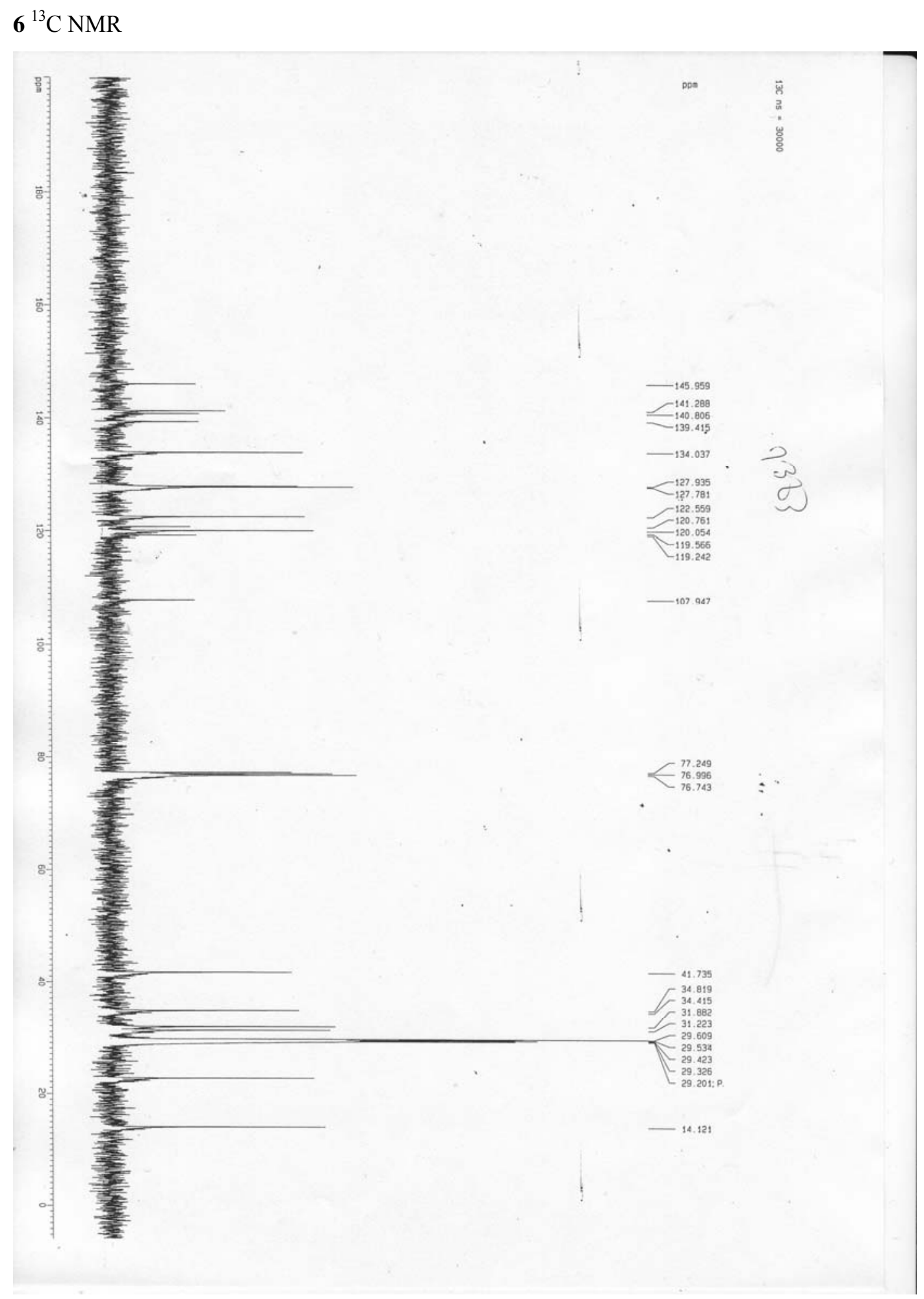




\section{$7{ }^{1} \mathrm{H}$ NMR}

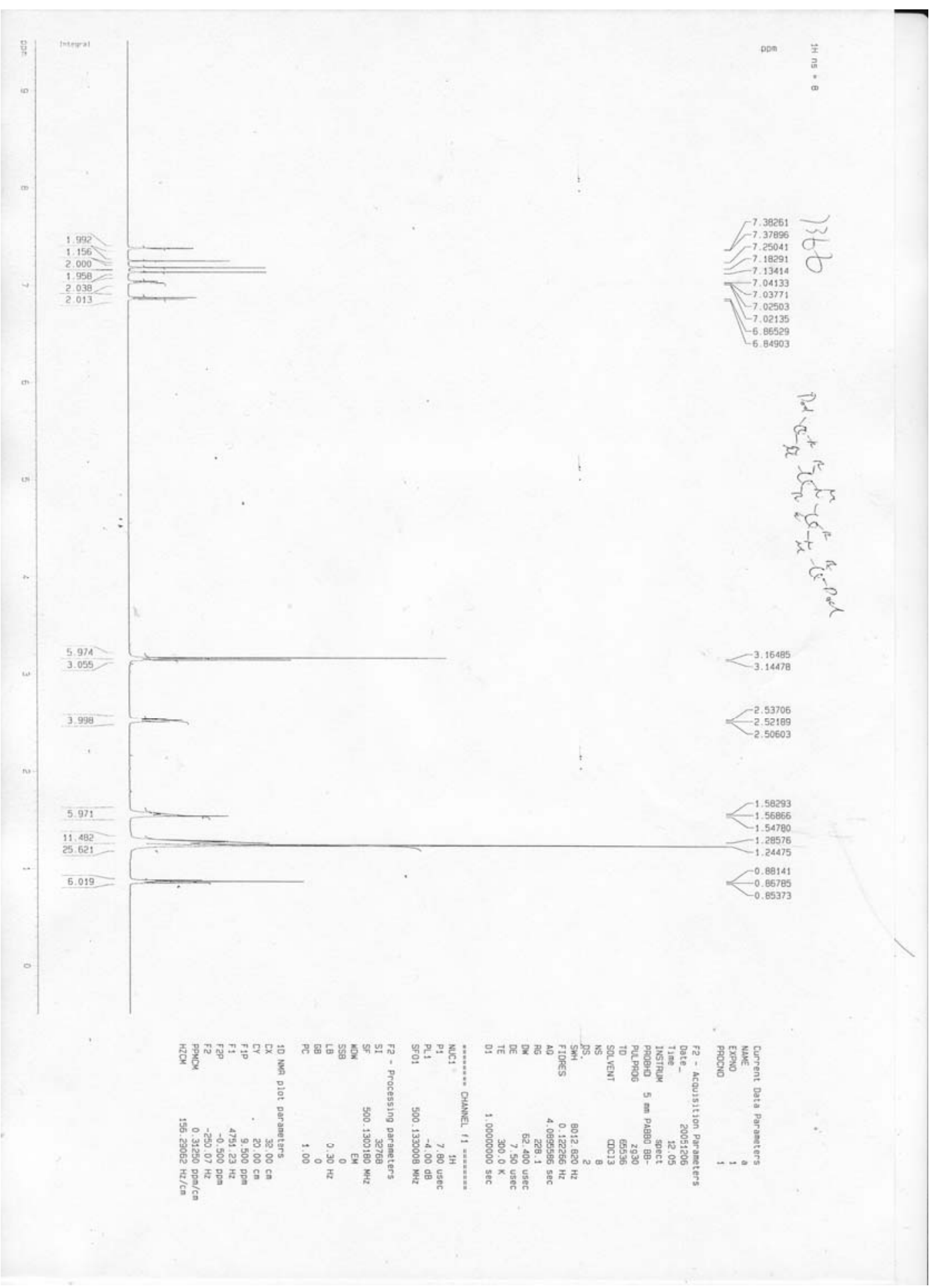


$7^{13} \mathrm{C}$ NMR

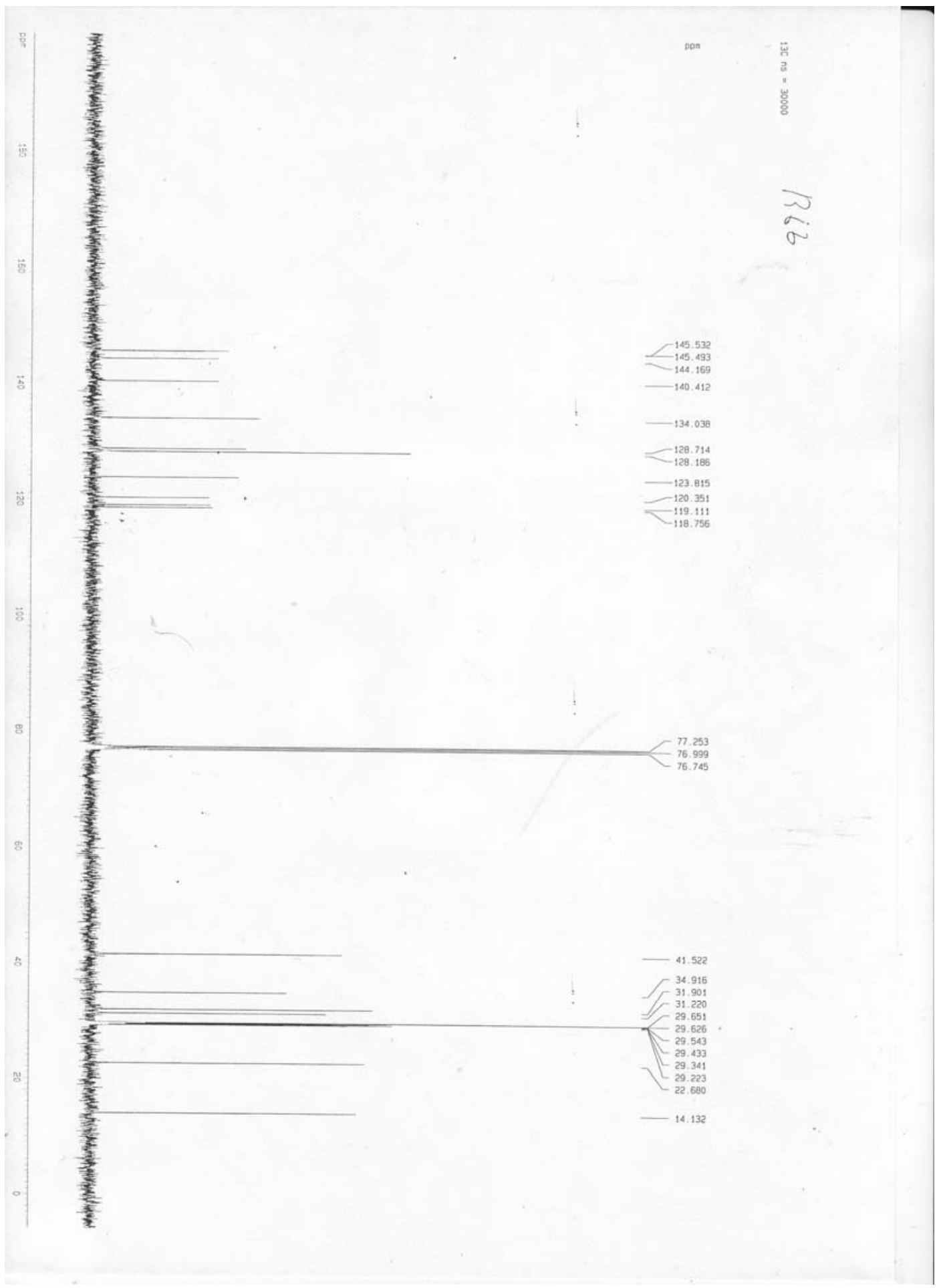




\section{$8{ }^{1} \mathrm{H}$ NMR}

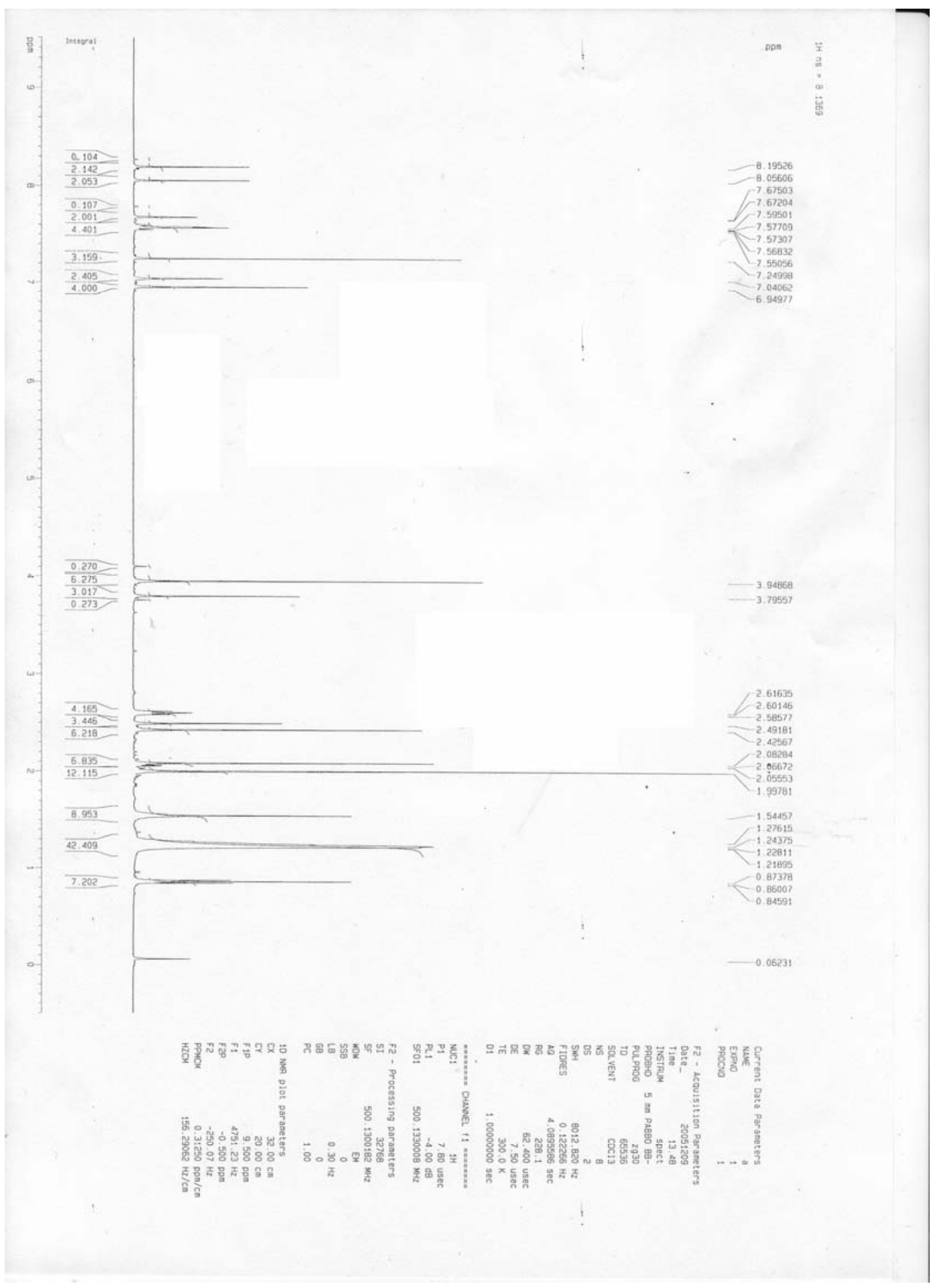




\section{$8{ }^{13} \mathrm{C}$ NMR}

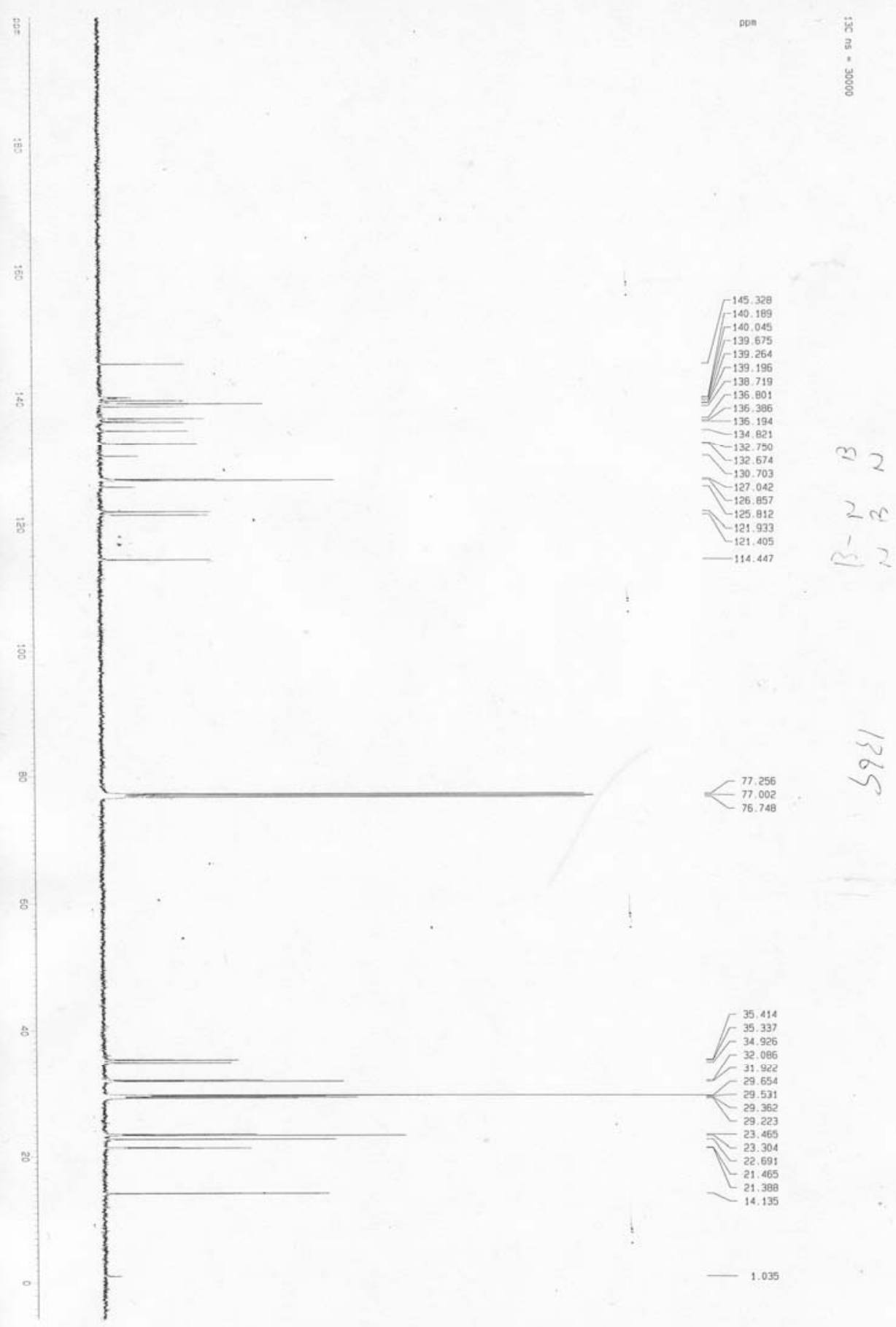




\section{$9{ }^{1} \mathrm{H}$ NMR}

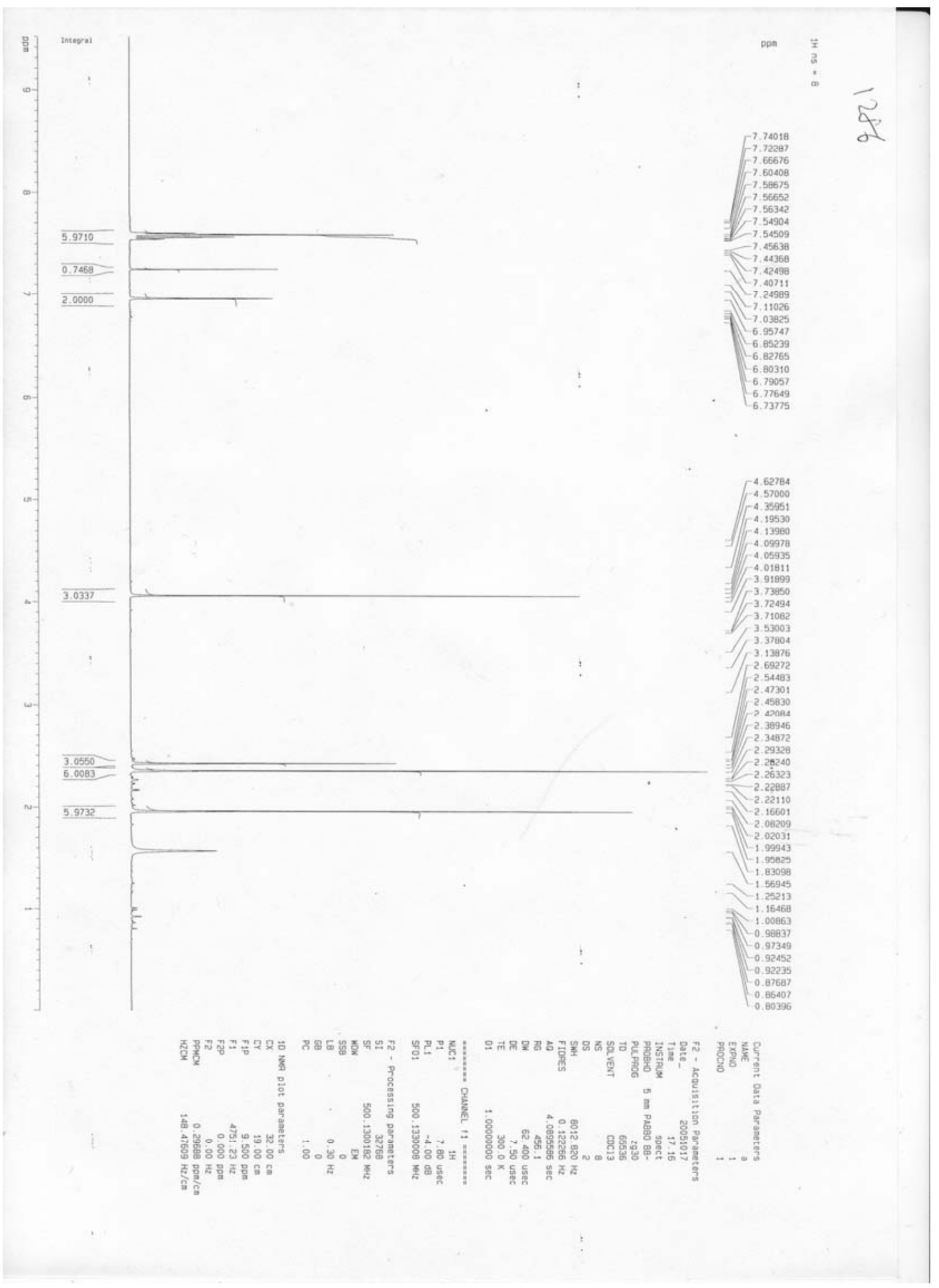


$9{ }^{13} \mathrm{C}$ NMR

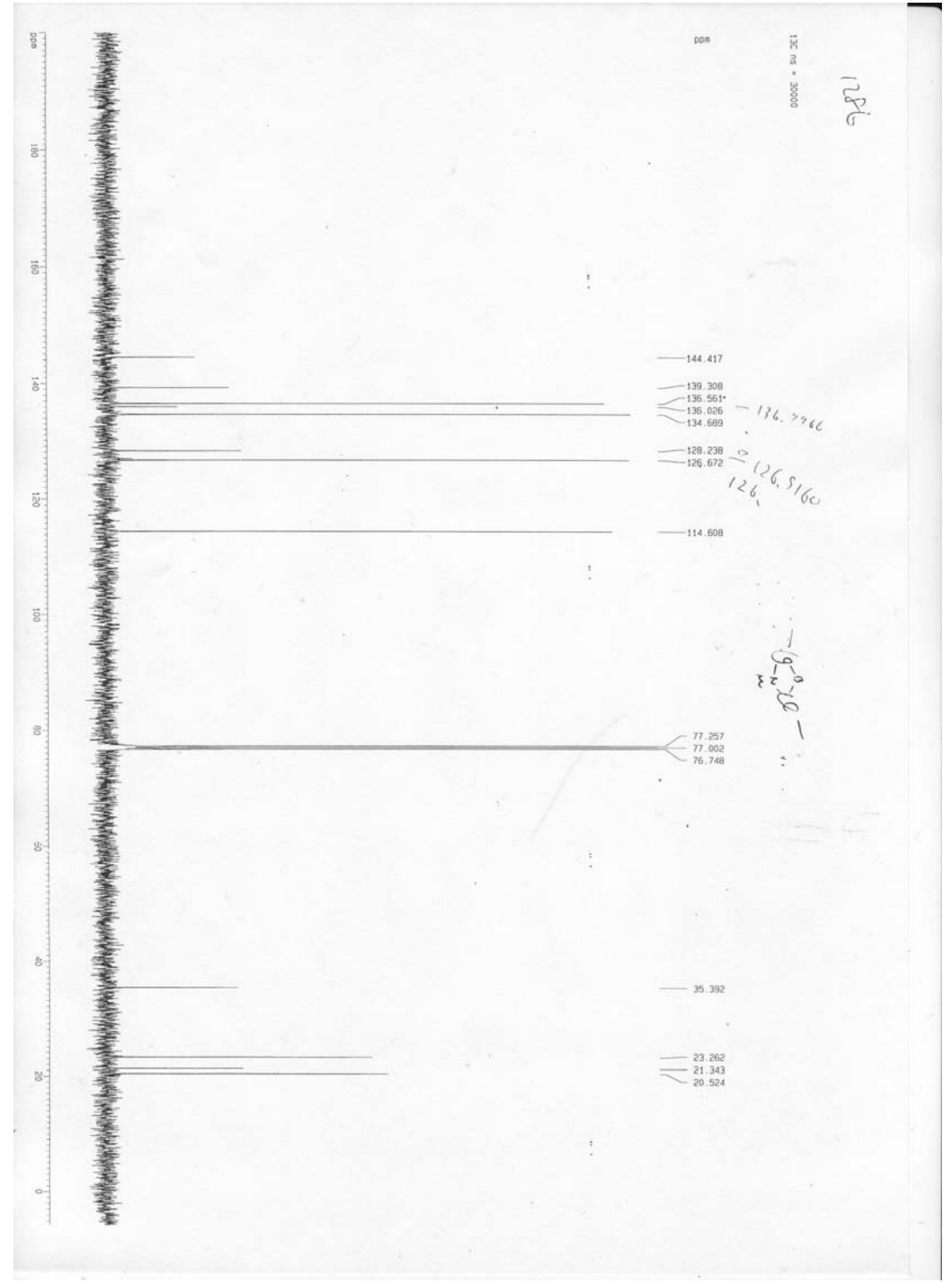

